\title{
Endogenous Growth, the Distribution of Wealth, and Optimal Policy under Incomplete Markets and Idiosyncratic Risk
}

Christiane Clemens

Maik Heinemann 


\title{
Endogenous Growth, the Distribution of Wealth, and Optimal Policy under Incomplete Markets and Idiosyncratic Risk ${ }^{\dagger}$
}

\author{
Christiane Clemens* \\ Bielefeld University \\ and CESifo
}

\author{
Maik Heinemann** \\ University of Potsdam
}

December 30, 2012

\begin{abstract}
This paper combines the Aiyagari/Huggett-type standard incomplete markets model with the Arrow/Romer approach to growth to analyze feedback effects between growth and inequality, both endogenously determined in equilibrium. We derive conditions on existence/ nonexistence of balanced growth paths. Major results include that growth, inequality, and risk are positively related in our model, but we also identify a hump-shaped relationship between welfare and risk, indicating a tradeoff relationship between risk-pooling and growth in the determination of welfare. We discuss transitory dynamics and policy implications. A growth policy simultaneously reduces wealth inequality in the economy. The benefits and burdens of the underlying policy are unequally distributed, which raises the issue of politico-economic equilibria. We provide results on majority voting, finding that that the median voter prefers less than optimal subsidies on investment. Interestingly, the society might even vote against a policy providing full insurance against idiosyncratic risk, because welfare losses of lower growth more than offset welfare gains from lower risk.
\end{abstract}

Keywords: endogenous growth, wealth distribution, idiosyncratic risk, borrowing constraints, heterogeneous agents, optimal policy

JEL classification: O4, D3, D8, D9, C6

\footnotetext{
$\dagger$ The simulations were run on a Linux cluster at RRZN at Leibniz University, Hannover, Germany. The authors would like to thank the cluster team for their support. This research is part of DFG priority program 1578 on 'Financial Market Imperfections and Macroeconomic Performance'. The authors would like to thank DFG for financial support. Discussion and helpful suggestions by seminar participants at the Universities of Mannheim, Karlsruhe, and Berlin, and at the 2012 summer meeting of the Econometric Society in Malaga is gratefully acknowledged.

*Bielefeld University, Germany, christiane.clemens@uni-bielefeld.de

**University of Potsdam, Germany, maik.heinemann@uni-potsdam.de
} 


\section{Introduction}

The question of how growth and inequality are related has been a matter of profound interest for many economists over the last decades. The relation is considered to be an ambivalent one, the traditional view emphasizing the famous 'equity vs efficiency' tradeoff, according to which greater income or wealth equality creates disincentives which prove harmful to growth. Contrary, economic analysis has shown that in the presence of market imperfections inequality actually may reduce the long-run growth rate of the economy such that redistributive policies are rendered desirable. ${ }^{1}$

In this paper, we present a simple model of individual risk, growth, and inequality. A special feature of our approach is that both, the growth rate as well as the distribution of income and wealth are endogenously determined in equilibrium and mutually dependent. We are therefore able to study possible feedback effects to gain a deeper understanding of the implications regarding the redistributive and growth consequences of public policy. Market imperfections enter in our story twofold, firstly, by the absence of risk-sharing arrangements, and secondly, by the presence of individual borrowing constraints.

We combine the simple growth mechanism introduced by Romer (1986) with the neoclassical standard incomplete markets model with idiosyncratic risks and borrowing constraints in the spirit of Huggett (1993) and Aiyagari (1994, 1995). The agents' income and wealth heterogeneity stems from serially correlated uninsurable shocks to labor efficiency. Households are subject to borrowing constraints, restricting their means to smooth the intertemporal consumption flow.

Our analysis focuses on three aspects. We provide necessary and sufficient conditions for the existence of a balanced growth path and demonstrate that these not necessarily have to be met, the results crucially depending on the degree of risk aversion. Hence, for particular parametric specifications of the model an equilibrium growth path might not exist. $^{2}$ Similar to Aiyagari and McGrattan (1998) and Japelli and Pagano (1999) feasible equilibria in our model economy may also be characterized by a growth rate larger than the equilibrium interest rate. Additionally, due to the mutual dependency between growth and inequality, the model features transitional dynamics. Our numerical simulations show that for both variables transition towards their stationary values is non-monotonic.

Our second focus lies on role of market imperfections for the determination of growth and inequality. We find that the presence of risk and borrowing constraints unambiguously has a positive effect on the long-run growth rate of the economy. Aggregate savings are larger compared to the complete markets economy because of the desire of risk averse individuals to protect themselves against fluctuations in their intertemporal consumption path and the limitation of not being able to borrow, a phenomenon which is well-known from the literature and referred to as 'buffer stock saving' (Carroll, 1997). From this follows naturally that a rise in idiosyncratic risk not only increases equilibrium inequality

\footnotetext{
${ }^{1}$ See the survey by Aghion et al. (1999) and references therein.

${ }^{2}$ To this end, our analysis completes the descriptive analysis of Bertola et al. (2006, ch. 9.3).
} 
but also the growth rate of the economy. Tightening credit constraints raises growth but has an equalizing effect on the wealth distribution, the latter also originating from the accumulation channel.

Our last focus is on the policy implications which follow from the underlying model. Endogenous growth results from externalities in human capital accumulation. This endogenous growth mechanism is known to generate allocations which fail Pareto-efficiency, therefore calling for policy intervention. We pursue this line by analyzing the effects of the prototypical policy recommendation for Romer (1986)-type endogenous growth models, which is subsidizing capital accumulation financed from a non-distortionary consumption tax in order to close the wedge between the private and the social return to capital.

Our numerical simulations come up with some interesting results for the interaction between growth, risk, and inequality. First of all, we do not generally observe the 'equity $v s$ efficiency' tradeoff. A growth policy aimed at improving efficiency of the underlying allocation simultaneously contributes to the equity goal by also lowering wealth inequality. Moreover, the policy under consideration lowers the riskiness of disposable income, such that feedback effects on growth additionally have to be taken into account. Finally, the consumption tax under consideration tends to be regressive as the underlying model features wealth and savings rate heterogeneity.

Wealth heterogeneity turns out to be crucial, when it comes to the evaluation of welfare effects. For both issues addressed, changes in risk as well as policy intervention, welfare gains and losses are unequally distributed across the society, closely relating our analysis to Domeij and Heathcote (2004) and Heathcote (2005) and also calling for a discussion of political equilibria in a median voter context.

We develop a procedure enabling us to assess welfare consequences of changing environments also including the transitional dynamics of the underlying economy towards a new steady state. Usually one would expect welfare gains from either, larger growth and lower risk. In our model, however, lower risk goes along with a disincentive to save out of precautionary motives. This leads to a decline in the growth rate and generates associated welfare losses. We illustrate this idea by performing a thought experiment on a redistributive policy aimed at completely eliminating the individual risk. Because this stands at odds with the growth target it might turn out welfare-deteriorating in the whole and also would be voted down by a majority of the population. Depending on the magnitude of risk, growth and risk-pooling effects are offsetting each other, such that we observe an inverted $U$-shaped relationship between welfare and risk.

Regarding the welfare consequences of the growth policy under consideration, a policy maximizing aggregate welfare is characterized by smaller degree of subsidization compared to the complete markets economy. This again, because policy benefits and burdens are unequally distributed over agents. We find that the median voter also prefers lower subsidies on investment, a point which was already raised by Bertola (1993) for the case of an exogenously given time-invariant wealth distribution. 
Our analysis relates to the literature in several ways. In the tradition of Galor and Zeira (1993), Banerjee and Newman (1993), or Bénabou (1996, 2000), we stress the importance of risk and market imperfections. Integrating the analysis of growth and inequality regularly suffers from technical drawbacks. Generally, it is difficult to jointly and endogenously determine both, the balanced growth rate and the equilibrium income and wealth distribution. Either assumptions have to be restrictive to prevent models from eluding closed-form solutions or one has to rely on numerical simulations, which is done in the present paper.

Previous work along this line, simultaneously and endogenously determining equilibrium inequality and the growth rate of the economy, is rare. Aiyagari (1994) only shortly refers to the possibility of including exogenous technical progress in the analysis (see Aiyagari, 1994, fn. 26) and pursues this in Aiyagari and McGrattan (1998). Japelli and Pagano $(1994,1999)$ discuss the implications of liquidity constraints on endogenous growth and welfare but do not consider distributional consequences. Bertola (1993) assumes an exogenously given, time-invariant wealth distribution in his discussion of the politico-economic implications of public policy in an endogenous growth context. Bertola et al. (2006) briefly raise the issue of endogenous growth in their discussion of the standard incomplete markets model. They neither provide existence results on equilibrium growth paths nor an numerical assessment of the feedback effects between risk, growth, inequality and borrowing constraints.

Perhaps closest to our approach, although with a different focus, is Krebs $(2003 a, b)$, who considers human capital formation in an heterogeneous agent model with idiosyncratic risk. He analyzes trade-off relationships between the two accumulated assets, real and human capital, and finds a risk effect on growth which is rather large in magnitude.

Our paper is organized as follows. We develop the model in section 2. For reference, we first give a short sketch of the equilibrium allocation in the representative agent complete markets economy before proceeding to the heterogeneous agent incomplete markets setting. We determine the macroeconomic equilibrium, the stationary wealth distribution, and state conditions on the existence of a balanced growth path. Section 3 is devoted to the numerical analysis. We start with a description of calibration procedures, examine the effects of a rise in idiosyncratic risk, shortly discuss the growth and welfare effects of providing full insurance against the idiosyncratic risk, and conclude the growth and distributional implications of changes in debt limits. Section 4 presents the policy analysis. The section covers the design of an optimal growth policy and deals with political economy issues by determining the politico-economic equilibrium. Section 5 concludes. 


\section{The Model}

\subsection{Overview: Equilibrium Growth and Complete Markets}

We consider a production function with knowledge spillovers in the spirit of Romer (1986). The labor force (population) is normalized to unity and there is no population growth. We consider a continuum $j \in[0,1]$ of identical firms who produce a homogeneous output good $y_{j, t}$ according to the following Cobb-Douglas technology :

$$
y_{j, t}=B k_{j, t}^{\alpha} l_{j, t}^{1-\alpha} K_{t}^{1-\alpha}
$$

where $B>0, \alpha \in(0,1)$, both constant. Capital depreciates at the constant rate $\delta>0$. Output is assumed to be generated from physical capital $k_{j, t}$ and labor $l_{j, t}$. If the individual producer expands $k_{j, t}$ by investment, the aggregate capital stock $K_{t}$ rises accordingly and generates a positive externality by raising the productivity of all firms. The production function of the individual firm is homogenous of degree one with respect to the privatelyowned inputs and twice continuously differentiable. Aggregate production is linear in the aggregate capital stock $K$ and displays increasing returns to scale, such that the requirements for ongoing growth of per capita incomes are met.

The optimization problem of the individual firm is standard. All markets are competitive, and factor prices are determined by the usual marginal productivity conditions. In market equilibrium $k_{j, t}$ equals $K_{t}$, when additionally considering the normalization of the labor force. The equilibrium real interest rate is determined by the private marginal product of capital and falls short of the social return, because the productivity-enhancing effect of investments is not taken into account in individual profit maximization. The equilibrium private gross interest rate $R=1+r$ is time-invariant and given by:

$$
R=\alpha B+1-\delta
$$

The equilibrium wage rate equals marginal labor productivity and grows proportionally to the aggregate capital stock:

$$
w_{t}=(1-\alpha) B K_{t} .
$$

Consumers are homogenous in an economy with complete markets, due to the possibility of trading state-contingent securities which allow for perfect risk-sharing. The infinitelylived representative agent maximizes discounted intertemporal utility from consumption, $c$, subject to the intertemporal budget constraint describing the dynamics of individual wealth holdings $a$ and taking prices as given:

$$
\max _{\left\{c_{t}\right\}_{t=0}^{\infty},\left\{a_{t+1}\right\}_{t=0}^{\infty}} V_{t}=\sum_{t=0}^{\infty} \beta^{t} u\left(c_{t}\right) \quad \text { s.t. } \quad a_{t+1}+c_{t}=R a_{t}+w_{t},
$$

where $0<\beta<1$ denotes the discount factor. Typically, the current period utility function displays constant relative risk aversion (constant IES), measured by the parameter $\rho>0$. 
Optimal consumption growth is determined by the usual Euler equation. The growth equilibrium in the economy with complete markets is characterized by factor and commodity market clearing. Individual asset holdings sum up to the aggregate capital stock. Output, consumption, and the capital stock grow at a common and constant equilibrium growth rate, $\gamma_{C}$, which follows as:

$$
1+\gamma_{C}=(\beta R)^{1 / \rho}
$$

The basic Romer (1986)-model displays no transitional dynamics. The economy immediately enters the balanced growth path. Due to the presence of technological spillovers, the equilibrium growth rate is suboptimally low in the decentralized economy if compared to the social optimum, which renders an appropriately chosen growth policy effective. Policy recommendations aim at subsidizing capital accumulation to close the wedge between the private and the social return to capital, with subsidies possibly being financed from a non-distortionary tax. In an economy with complete markets and no endogenous labor-leisure choice, taxing either consumption or labor income ultimately amounts to a lump-sum tax.

\subsection{Idiosyncratic Risks and Incomplete Markets}

Consider an economy populated by a continuum $[0,1]$ of infinitely-lived households who are ex ante identical and heterogeneous ex post. Each household is endowed with one unit of labor which he supplies inelastically to the labor market. In each period of time, the household is subject to an idiosyncratic shock to his labor productivity, which exposes him to a labor income risk. Markets are incomplete, such that the individual risk cannot be perfectly pooled (cf. Aiyagari, 1994, 1995). Households are risk-averse and can save or borrow in order to smooth their intertemporal consumption flows. Borrowing, however, is constrained up to a given limit. As a result, the agents self-insure by undertaking precautionary savings to build a buffer against future losses or drops in income. There is no aggregate risk and no risk on capital return.

Let $\theta_{i, t}$ denote agent $i$ 's labor productivity of period $t$. We assume that $\theta_{i, t}$ evolves according to a finite state first-order Markov-process with bounded support, lowest possible realization $\theta_{\min }$ such that $\theta_{i, t} \geqslant \theta_{\min } \geqslant 0, \mathrm{E}_{\theta}\left[\theta_{i, t}\right]=1$ for all $t$, and the associated probability transition matrix $P\left(\theta_{t}, \theta_{t+1}\right)=\operatorname{prob}\left(\theta_{t+1} \mid \theta_{t}\right)$, where the expectation is formed with respect to the stationary distribution of the underlying Markov process.

Let $a_{i, t}$ denote agent $i$ 's holdings of non-human wealth in period $t$. For given gross factor prices $R$ and $w_{t}$, the individual household's intertemporal budget constraint is given by:

$$
a_{i, t+1}+c_{i, t}=R a_{i, t}+w_{t} \theta_{i, t} .
$$

The borrowing constraint requires $a_{i, t} \geqslant-\phi_{t}$ for all $t$, where $\phi_{t}$ either might stand for the natural debt limit (Aiyagari, 1994, p. 666) or for some ad hoc limit. 
The household derives utility from streams of consumption $\left\{c_{i, t}\right\}_{t=0}^{\infty}$. Intertemporal preferences are time-separable. Assuming CRRA preferences, the intertemporal problem of agent $i$ can be set up as follows:

$$
\begin{aligned}
\max _{\left\{c_{i, t}\right\}_{t=0}^{\infty},\left\{a_{i, t+1}\right\}_{t=0}^{\infty}} \mathrm{E}_{t} \sum_{t=0}^{\infty} \beta^{t} \frac{c_{i, t}^{1-\rho}-1}{1-\rho} & \\
\text { s.t. } \quad a_{i, t+1}+c_{i, t} & =R a_{i, t}+w_{t} \theta_{i, t} \\
a_{i, t+1} & \geqslant-\phi_{t+1} .
\end{aligned}
$$

Note that, by (2) and (3), the equilibrium factor prices are completely determined by the underlying technology and do not depend on the wealth distribution. The labor income risk does not vanish in the long run, because the equilibrium wage rate, $w_{t}$, grows linearly in the capital stock. We generally assume that the borrowing limit, $\phi_{t}$, grows proportionally to the aggregate capital stock. Because the labor income risk is multiplicatively related to the wage rate, the borrowing constraint does not cease to be binding in the long-run in a growing economy.

In a next step, we transform problem (5) such that it corresponds to the associated problem of a stationary economy (cf. Aiyagari, 1994; Aiyagari and McGrattan, 1998). Let $\gamma$ denote the equilibrium growth rate of the incomplete markets economy. We use the transformations $\tilde{a}_{i, t}=a_{i, t} / K_{t}, \tilde{c}_{i, t}=c_{i, t} / K_{t}, \tilde{\phi}_{t}=\phi_{t} / K_{t}, \tilde{w}=w_{t} / K_{t}$ and $\tilde{\beta}=\beta(1+\gamma)^{1-\rho}$, where the aggregate capital stock, $K_{t}>0$, equals average capital holdings. The detrended constraint is time-invariant, that is $\tilde{\phi}_{t}=\tilde{\phi}$ for all $t$.

We are now able to write down the Bellman equation describing the agent's intertemporal problem: ${ }^{3}$

$$
\begin{array}{r}
V(\hat{a}, \theta)=\max _{\tilde{c}, \hat{a}^{\prime} \geqslant 0}\left\{u(\tilde{c})+\tilde{\beta} E^{\prime} V\left(\hat{a}^{\prime}, \theta^{\prime}\right)\right\} \\
\text { s.t. } \quad \hat{a}^{\prime}(1+\gamma)+\tilde{c}=R \hat{a}+[(1+\gamma)-R] \tilde{\phi}+\tilde{w} \theta, \quad \hat{a}^{\prime} \geqslant 0
\end{array}
$$

where $\hat{a}=\tilde{a}+\tilde{\phi}$ is defined to include the borrowing constraint and primes denote variables of the next period.

In order to rule out Ponzi games, it is necessary to impose an ad hoc limit $\tilde{\phi}_{a h} \geqslant 0$ for the case of $R \leqslant 1+\gamma$. For the opposite case of $R>1+\gamma$, the optimization problem (6) implies that no agent will ever go into debt beyond the so-called 'natural debt limit', $\tilde{\phi}_{n}$, which is given by the worst-case scenario discounted value of labor income allowing (a.s.) for nonnegative consumption levels, that is

$$
\tilde{\phi}_{n}=\frac{\tilde{w} \theta_{\min }}{R-(1+\gamma)} .
$$

An arbitrarily fixed ad hoc debt limit $\tilde{\phi}_{a h}$ will only be binding if $\tilde{\phi}_{a h} \leqslant \tilde{\phi}_{n}$. Therefore, the effective debt limit appearing in the optimization problem (6) below is implicitly

\footnotetext{
${ }^{3}$ For notational convenience, we drop the explicit time notation and the index $i$ related to individual decisions.
} 
determined by the wage rate, the interest rate, and the growth rate $\gamma$, all to be determined in equilibrium

$$
\tilde{\phi}_{a h} \geqslant 0, \quad \tilde{\phi}= \begin{cases}\tilde{\phi}_{a h}, & R \leqslant 1+\gamma \\ \min \left[\tilde{\phi}_{a h}, \tilde{\phi}_{n}\right], & R>1+\gamma .\end{cases}
$$

For $\tilde{\beta}<1$, the optimization problem (6) is bounded and structurally equivalent to the one discussed in Aiyagari (1994). It is, however, important to bear in mind that $\tilde{\beta}$ crucially depends on $\rho$ and $\gamma$. The condition $\tilde{\beta}<1$ is not trivially satisfied by assuming a time discount factor $\beta$ in the unit interval. ${ }^{4}$ We will return to this issue when discussing existence and feasibility of a balanced growth path.

Let $\hat{a}^{\prime}=h(\hat{a}, \theta ; \gamma, \tilde{\phi}, \tilde{w}, R)$ denote the associated policy function solving problem (6). Following Aiyagari (1994), we employ the individual policy functions to derive aggregate (average) wealth holdings. Given the properties of the stochastic process underlying the idiosyncratic shocks to labor efficiency, the stationary distribution of wealth levels $\hat{a}$ across agents is represented by the stationary probability measure $\mu(\hat{a}, \theta ; \gamma, \tilde{\phi}, \tilde{w}, R)$, such that aggregate detrended wealth holdings are given by:

$$
\mathrm{E}_{\mu} h(\hat{a}, \theta ; \gamma, \tilde{\phi}, \tilde{w}, R) \equiv \hat{A}(\gamma, \tilde{\phi}, \tilde{w}, R)=\tilde{A}(\gamma, \tilde{\phi}, \tilde{w}, R)+\tilde{\phi}
$$

where $\mathrm{E}_{\mu}$ denotes the expectation with respect to the stationary distribution. Recall that the equilibrium factor prices $\tilde{w}$ and $R$ do not depend on the wealth distribution, due to the properties of the production technology (1).

\subsection{Balanced Growth and General Equilibrium}

Balanced growth in the Romer (1986)-economy is characterized by consumption, output and the capital stock growing at a common rate. The equilibrium growth rate is constant along the balanced growth path due to the time-invariant capital return. The production of aggregate output is nonstochastic, but indirectly affected by individual saving decisions via the aggregate capital stock. The equilibrium factor prices coincide with the expressions (2) and (3) derived for the representative agent economy.

Given the production technology (1), profit maximization implies the following factor demands of the individual firm, which we express as functions of the detrended variables $\tilde{w}_{t}=w_{t} / K_{t}$ and $\tilde{k}_{j, t}=k_{j, t} / K_{t}$ for notational convenience:

$$
l_{j, t}=\left(\frac{\tilde{w}_{t}}{(1-\alpha) B}\right)^{-1 / \alpha} \tilde{k}_{j, t} \quad \text { and } \quad \tilde{k}_{j, t}=\left(\frac{R_{t}-1+\delta}{\alpha B}\right)^{-1 /(1-\alpha)} l_{j, t} .
$$

\footnotetext{
${ }^{4}$ This is a standard result in intertemporal modeling and usually covered by the transversality condition in representative agent economies, which rules out unlimited borrowing and unbounded welfare. Ponzi games are ruled out in the present model by (8). For existence of a balanced growth, see Proposition 1 below.
} 
Aggregating individual factor demands yields:

$$
\begin{aligned}
& \int_{0}^{1} l_{j, t} \mathrm{~d} j \equiv L\left(R_{t}, \tilde{w}_{t}\right)=\left(\frac{\tilde{w}_{t}}{(1-\alpha) B}\right)^{-1 / \alpha} \tilde{K}\left(R_{t}, \tilde{w}_{t}\right) \\
& \int_{0}^{1} \tilde{k}_{j, t} \mathrm{~d} j \equiv \tilde{K}\left(R_{t}, \tilde{w}_{t}\right)=\left(\frac{R_{t}-1+\delta}{\alpha B}\right)^{-1 /(1-\alpha)} L\left(R_{t}, \tilde{w}_{t}\right) .
\end{aligned}
$$

Definition 1 A decentralized recursive competitive stationary equilibrium in detrended variables is defined by a detrended wage rate $\tilde{w}$, a gross interest rate $R$, a growth rate $\gamma$, and a policy function $h(\hat{a}, \theta ; \gamma, \tilde{\phi}, \tilde{w}, R)$ with associated value function $V(\hat{a}, \theta)$ such that the following conditions hold:

(i) The policy function $h(\hat{a}, \theta ; \gamma, \tilde{\phi}, \tilde{w}, R)$ solves the consumer's optimization problem (6), such that the aggregate detrended asset supply is given by $\tilde{A}(\gamma, \tilde{\phi}, \tilde{w}, R)$.

(ii) The economy-wide capital stock $K_{t}$ equals the aggregation of firm-specific capital stocks, which in detrended variables is equivalent to

$$
\tilde{K}(R, \tilde{w})=\int_{0}^{1} \frac{k_{j, t}}{K_{t}} \mathrm{~d} j=1 .
$$

(iii) Factor markets clear at given (detrended) prices $\tilde{w}=(1-\alpha) B$ and $R=\alpha B+1-\delta$ and aggregate detrended factor employment is given by

$$
\begin{aligned}
L(R, \tilde{w}) & =\mathrm{E}_{\theta}\left[\theta_{i, t}\right]=1 \\
\tilde{K}(R, \tilde{w}) & =\tilde{A}(\gamma, \tilde{\phi}, \tilde{w}, R) .
\end{aligned}
$$

Capital demand equals aggregate wealth holdings. In detrended variables, this is equivalent to

$$
\tilde{A}(\gamma, \tilde{\phi}, \tilde{w}, R)=1 \Longleftrightarrow \hat{A}(\gamma, \tilde{\phi}, \tilde{w}, R)=1+\tilde{\phi}
$$

(iv) The stationary distribution $\mu(\hat{a}, \theta ; \gamma, \tilde{\phi}, \tilde{w}, R)$ of agents over individual wealth holdings and associated productivities is the fixed point of the law of motion which is consistent with the individual decision rules and equilibrium prices.

The equilibrium growth rate, consistent with a balanced growth path, is implicitly defined by (13).

Proposition 1 A unique balanced growth path with growth rate $\gamma$ exists in the presence of idiosyncratic shocks and incomplete markets, if $\beta<R^{\rho-1}$.

1. The necessary condition is sufficient

(i) for all $\rho \geqslant 1$,

(ii) for $\rho<1$, if the natural debt limit $\tilde{\phi}_{n}$ applies. 
Figure 1: Equilibrium growth rate, borrowing limits, and asset supply

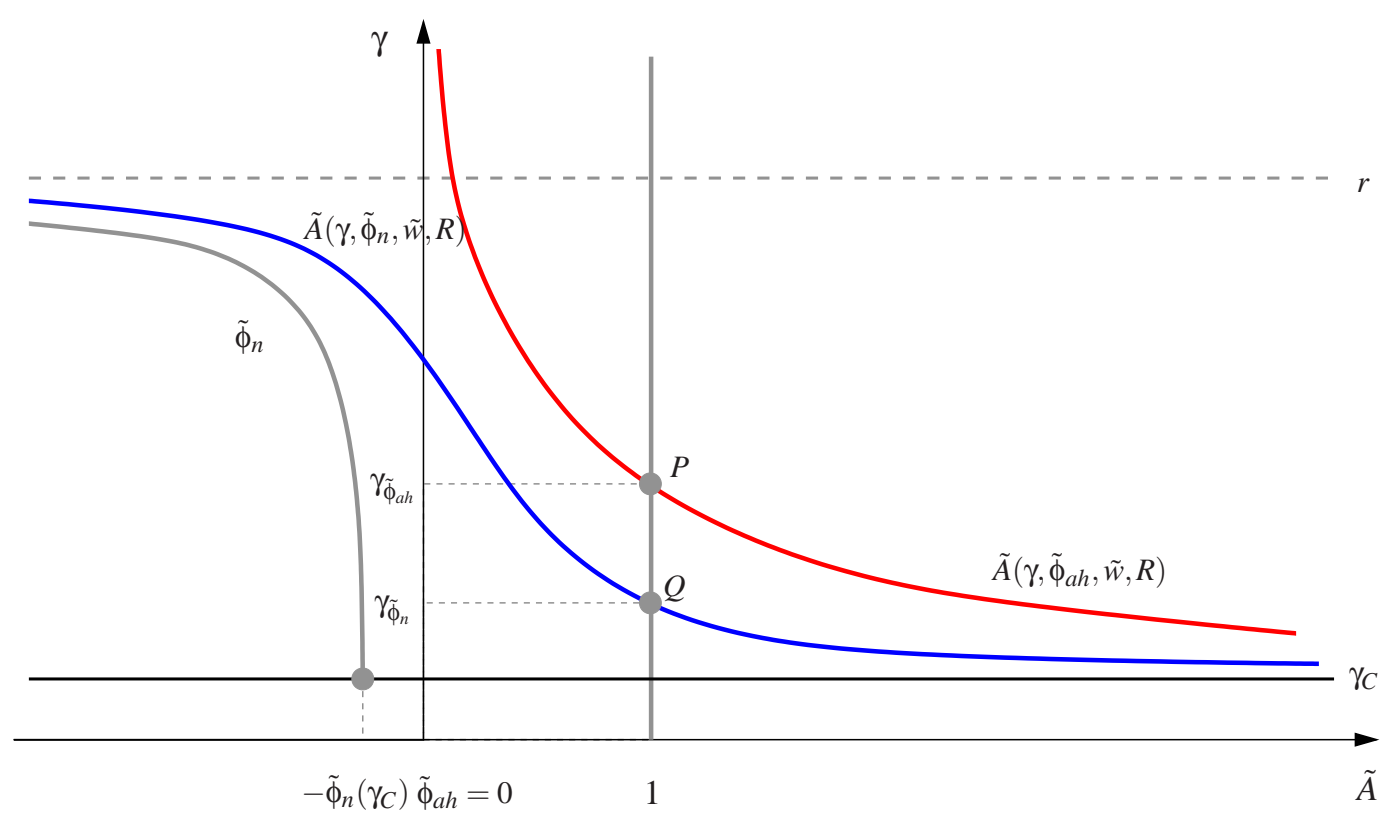

2. The equilibrium growth rate $\gamma$ is always larger than the associated growth rate of the economy with complete markets, that is $\gamma>\gamma_{C}$. The equilibrium growth rate is strictly smaller than the equilibrium interest rate, that is $R-1>\gamma>\gamma_{C}$, if the natural debt limit $\tilde{\phi}_{n}$ applies.

Proof of 1. and 2. see Appendix A.1.

Aiyagari (1994) proves existence for the case of CRRA utility and iid income. We are not able provide a proof for the more general model with serially correlated shocks, but verify existence in our numerical work.

A major implication of Proposition 1 is that a balanced growth path might not exist for an arbitrarily chosen ad hoc limit, $\tilde{\phi}_{a h}$, if the degree of risk aversion is sufficiently small. Appendix A.2 gives an example for this case. Another implication is that for some given ad hoc debt limit the equilibrium growth rate may well exceed the equilibrium interest rate, implying dynamic inefficiency (see also Aiyagari and McGrattan, 1998).

We find that the presence of uninsurable idiosyncratic labor income risk has an unambiguously positive effect on the aggregate growth rate of the economy by raising capital accumulation due to precautionary motives. This extends the results well-known from the standard (stationary) incomplete markets model to an endogenous growth context. Existence of a balanced growth path is tied to restrictions on the real interest rate and the intertemporal discount rate which closely resemble the key property $\beta R<1$ for existence of an equilibrium in stationary Bewley (1983)/Aiyagari (1994)-type models.

To illustrate the analogy of results, consider Figure 1 which plots the growth rate against aggregate (detrended) asset supply $\tilde{A}$ for both, some ad hoc debt limit $\tilde{\phi}_{a h}=0$ 
(red) and the natural debt limit $\tilde{\phi}_{n}$ (blue) as defined in (7). The figure also depicts $\tilde{\phi}_{n}$ as endogenously determined lower natural bound to asset holdings (grey). From (8) we know that the effective debt limit is determined by the size of the interest rate relative to the growth rate. Figure 1 also shows that any ad hoc debt limit, $\tilde{\phi}_{a h}$, smaller than the natural debt limit at the lowest possible growth rate, $\tilde{\phi}_{n}\left(\gamma_{C}\right)$, sustaining non-negative consumption in the incomplete markets economy definitely is binding for all $\gamma>\gamma_{C}$. Obviously, any ad hoc debt limit acts more restrictive on intertemporal consumption smoothing than the natural debt limit, resulting in relatively increased asset holdings and a comparably larger equilibrium growth rate $\gamma_{\tilde{\phi}_{a h}}>\gamma_{\tilde{\phi}_{n}}$; the respective equilibria for the two debt limits are represented by $P$ and $Q$ in Figure 1 .

The existence condition $\beta R<1$ for stationary economies modifies to $\beta R<(1+\gamma)^{\rho}$ in a growing incomplete markets economy, which is equivalent to $\tilde{\beta} R<1+\gamma^{5}$ Hence, we have a result similar to Aiyagari (1994) that wealth holdings grow to infinity, if the interest rate approaches the (growth-adjusted) discount rate from below, or, likewise, if $1+\gamma$ approaches $\tilde{\beta} R$ from above. Consequently, the growth rate of the complete markets economy, $\gamma_{C}$, where in equilibrium $1+\gamma_{C}=\tilde{\beta} R$, constitutes a lower bound for a balanced growth path.

Feasibility of the allocation also requires lifetime utility to be bounded, i.e. $\tilde{\beta}<1$. This condition is satisfied if $\beta<R^{\rho-1}$, which is the condition given in Proposition 1 . As can be seen, the requirements for bounded welfare are trivially met for any $\rho \geqslant 1$. For the case of $\rho<1$, the restriction $\tilde{\beta}<1$ imposes an upper bound on feasible growth rates, such that $\beta<R^{\rho-1}$ constitutes a necessary condition.

The aggregate growth rate is non-stochastic. All households experience identical consumption and income growth, although heterogeneity prevails, because the labor income risk does not vanish in the long run. Individual wealth levels grow at the constant rate $\gamma$ and are distributed in accordance with the limiting distribution of the labor productivity shocks, which is stationary in the detrended variables.

\section{Numerical Simulations}

\subsection{Calibration}

In what follows, we want to provide a numerical assessment of the growth and welfare of effects a change in risk and of the tax-subsidy scheme of section 4. Regarding the parameters related to preferences and technology, we use standard calibrations from the literature. The production technology is Cobb-Douglas. We set the capital share to 0.33 , which is in line with estimates of Prescott (1986). Capital depreciation is fixed at 0.08 . We set the discount factor $\beta$ to 0.985 and simulate our model for different values of the coefficient of relative risk aversion $\rho$ ranging from 1.5 to 5 . The productivity parameter $B$ is a free parameter and set to 0.33 , which is chosen to target an annual equilibrium

\footnotetext{
${ }^{5}$ See the proof of Proposition 1 in the Appendix and footnote 26 in Aiyagari (1994).
} 
Table 1: Numerical specification of the baseline model

\begin{tabular}{cl|c}
\hline \hline parameter & & calibration target \\
\hline \hline$\alpha$ & 0.33 & capital share $=0.33$ \\
$\delta$ & 0.08 & \\
$B$ & $0 . \overline{33}$ & $r_{C}=0.03$ \\
\hline$\rho$ & $1.5,3,5$ & $\gamma_{C}=0.0048$ \\
$\beta$ & 0.985 & \\
\hline$\sigma_{\varepsilon}^{2}$ & 0.0171 & $\operatorname{Var}[\ln (w \theta)]=0.09$ \\
$\rho_{\theta}$ & 0.9 & $\rho_{\ln (w \theta)}=0.9$ \\
\hline$\tilde{\phi}_{a h}$ & 0 & \\
\hline \hline
\end{tabular}

(riskless) real interest rate of around 3\%. We assume a lognormal $A R(1)$ process for labor efficiency with normalized mean, $E[\theta]=1$, which in continuous space is given by

$$
\ln \theta^{\prime}=-1 /\left(1+\rho_{\theta}\right) \frac{\sigma_{\varepsilon}^{2}}{2}+\rho_{\theta} \ln \theta+\varepsilon,
$$

where $\varepsilon \sim \mathcal{N}\left(0, \sigma_{\varepsilon}^{2}\right)$ and $\operatorname{Var}(\ln \theta)=\operatorname{Var}(\ln (\tilde{w} \theta))=\sigma_{\varepsilon}^{2} /\left(1-\rho_{\theta}^{2}\right)$. The stochastic process for the underlying labor productivity shocks is specified such as to display a serial correlation in $\log$ labor incomes of 0.9 and a standard deviation of 0.3 , which matches empirical evidence for the U.S. provided by Storesletten et al. (2004) or Guvenen (2009). The AR(1) process is approximated in discrete state-space by a five-state Markov chain using the method proposed by Rouwenhorst (1995). ${ }^{6}$ The implied income distribution also matches recent estimates for the actual distribution in the U.S., the simulations broadly generating Gini coefficients ranging between 0.3 and 0.4 .

The lognormal theoretical distribution underlying the labor income process implies a lowest possible realization for $\theta$ infinitesimally close to naught. By (7), a narrow interpretation of the model in our numerical simulations would also imply a zero natural debt limit. ${ }^{7}$ Taking account of this, most of our numerical simulations are based on assuming a zero debt limit ad hoc, that is $\tilde{\phi}_{a h}=0$. In the more general setting discussed in section 3.4, we relax this assumption. In order to explore the question of how debt limits affect long-run growth, we also allow for $\tilde{\phi}_{n} \geqslant 0$.

We report results for three alternative settings, (a) the complete markets economy (indicated by subscript $C$ ), (b) the case of an exogenously fixed ad hoc debt limit, where $\tilde{\phi}_{a h}=0$, and (c), in section 3.4 for the endogenously determined natural debt limit $\tilde{\phi}_{n}$. Table 1 reports the parameter values applied in our numerical simulations.

\footnotetext{
${ }^{6}$ As documented by Kopecky and Suen (2010), this method is more reliable than others for the approximation of highly persistent processes.

${ }^{7}$ The numerical simulations, of course, are capable of generating nonzero natural debt limits, as the stochastic process is simulated by a discrete five-state Markov chain.
} 


\subsection{Measuring Welfare}

Besides the growth and distributional effects of a change in risk or of the adoption of some public policy respectively, our aim is to qualitatively and quantitatively assess the associated welfare consequences. To this end, we employ a utilitarian welfare measure, aggregating individual welfare gains (or losses) over all agents in the economy (cf. Aiyagari and McGrattan, 1998). The welfare measure is consumption-related, computing the aggregate amount of consumption necessary to leave each consumer indifferent between the original allocation vis-à-vis the allocation resulting from a change in parameters or from the introduction of some policy (i.e. the compensating variation).

Changes in the general environment affect the equilibrium growth rate of the economy either directly, by immediate adjustments of factor prices to their new equilibrium values, or indirectly, via the associated response in the equilibrium wealth distribution. The latter triggers transitional dynamics, where the growth rate asymptotically approaches its new (and hence onward constant) equilibrium value. This constitutes a major difference to the complete markets endogenous growth model, where the economy immediately jumps onto the new steady-state following a change in conditions.

Our welfare measure accounts for the transitory adjustments. ${ }^{8}$ To this end, we develop a procedure allowing us to calculate the actual time path of the capital stock relative to an artificially constructed 'reference path'. The reference path mimics the identical long-run capital stock of the original economy under transitional dynamics, but posits instead that the economy has grown at the new equilibrium growth rate from the outset, while starting from a lower initial level; see Appendix B for a detailed description.

We calculate the change in consumption, $\Delta(\hat{a}, \theta) \times \tilde{c}(\hat{a}, \theta)$, for an individual with wealth $\hat{a}$ and productivity $\theta$ that equates the value function $V(\hat{a}, \theta)$ under the two different allocations (' 0 ' and ' 1 ') under consideration

$$
\Delta(\hat{a}, \theta)=K^{1}\left(\frac{V^{1}\left(\hat{a} / K^{1}, \theta\right)}{V^{0}(\hat{a}, \theta)}\right)^{\frac{1}{1-\rho}}-1,
$$

where the scaling factor $K^{1}$ corrects for the differences in initial capital stocks between the actual and the reference path. Consumption of an individual characterized by wealth $\hat{a}$ and productivity $\theta$ along the original path is given by

$$
\tilde{c}^{0}(\hat{a}, \theta)=R \hat{a}+[1+\gamma-R] \tilde{\phi}+\tilde{w} \theta-(1+\gamma) h^{0}(\hat{a}, \theta),
$$

where $h^{0}(\hat{a}, \theta)$ denotes the optimal policy function in the original situation. The amount $\Delta(\hat{a}, \theta) \tilde{c}^{0}(\hat{a}, \theta)$ leaves the consumer indifferent between the two allocations under comparison. We then employ the stationary probability measure $\mu^{0}$ to compute the aggregate

\footnotetext{
${ }^{8}$ Note that the transformed value function $V(\hat{a}, \theta)$ represents the solution to the optimization problem in stationarized variables. In order to determine lifetime utility we have to correct for the level of the capital stock.
} 


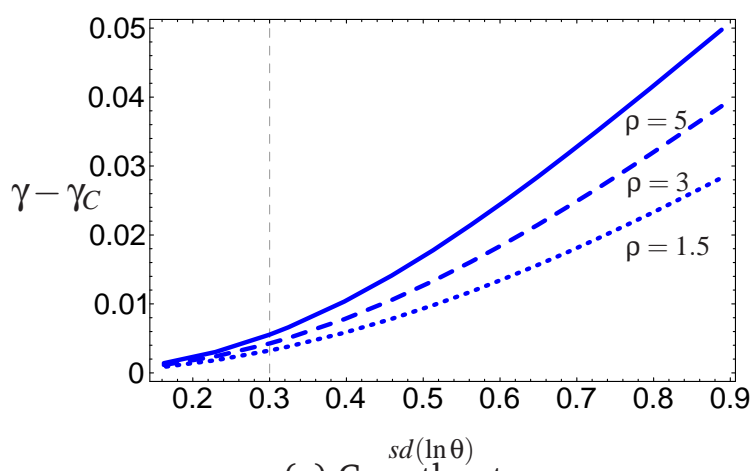

(a) Growth rate

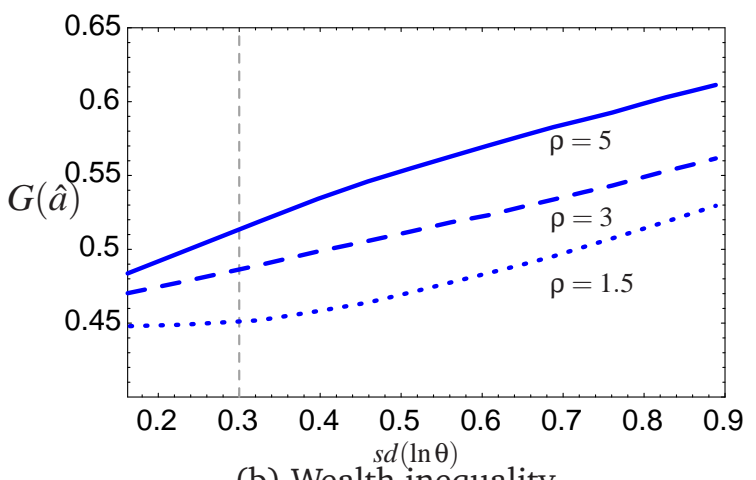

(b) Wealth inequality

Figure 2: Risk, growth, and inequality

percentage increase in consumption, $W$, leaving all agents indifferent

$$
W=\frac{\mathrm{E}_{\mu^{0}}\left[\Delta(\hat{a}, \theta) \tilde{c}^{0}(\hat{a}, \theta)\right]}{\mathrm{E}_{\mu^{0}}\left[\tilde{c}^{0}(\hat{a}, \theta)\right]} \times 100 .
$$

A positive value of $W$ identifies a welfare gain associated with moving from the original to the new allocation.

\subsection{Macroeconomic Effects of Rising Idiosyncratic Risk}

We now proceed with quantifying the macroeconomic effects of a mean preserving spread in shocks to labor efficiency. Our baseline specification assumes a standard variation of $\log$ labor incomes of $s d(\ln \theta)=0.3$; for reference see Table 1 . The results from our numerical simulations are summarized in Figures 2 and 4, which show the response of the equilibrium growth rate, wealth inequality, and welfare to an increase in the standard deviation of log labor incomes for three different degrees of risk aversion, $\rho=\{1.5,3,5\} .{ }^{9}$

Our numerical findings confirm the growth implications of uninsurable labor income risk stated in Proposition 1 that balanced growth in the presence of incomplete insurance markets and borrowing constraints exceeds growth in the complete markets economy due to precautionary motives. The long-run equilibrium growth rate rises monotonically with an increase in labor income risk, which reflects that households raise their savings in order to protect themselves against a higher earnings risk. Figure 2a illustrates this result by plotting the growth differential $\gamma-\gamma_{C}$ against the standard deviation of log labor incomes. The underlying relationship is positive and convex, meaning that the individual desire to self-insure by undertaking buffer-stock savings even gains importance for higher risk. The growth differential and therefore the growth effect of risk also is larger the more risk averse households are. Given the ad hoc debt limit of $\tilde{\phi}_{a h}=0$, the equilibrium growth

\footnotetext{
${ }^{9}$ We are primarily interested in the macroeconomic effects of a change in risk and therefore do not perform a full comparative static analysis with respect to the model primitives and only report results for three alternative values of $\rho$. Assuming non-expected utility generates the well-known results, that the growth rate increases with a rise in risk aversion and declines with a rise in the IES.
} 


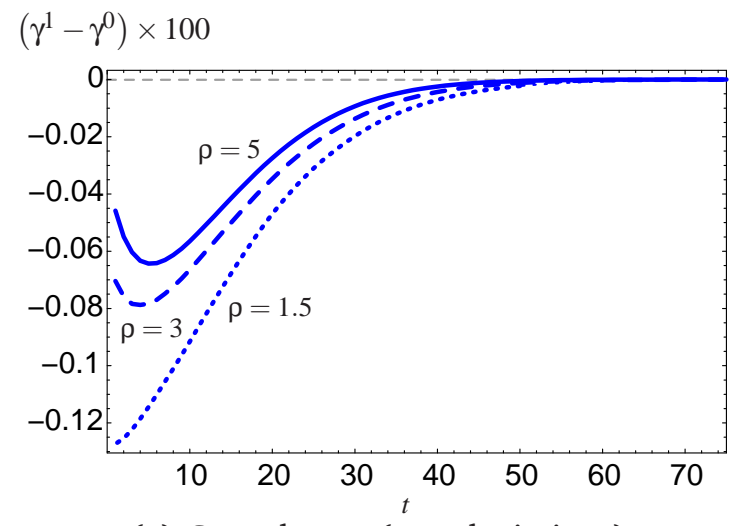

(a) Growth rate (p.p. deviations)

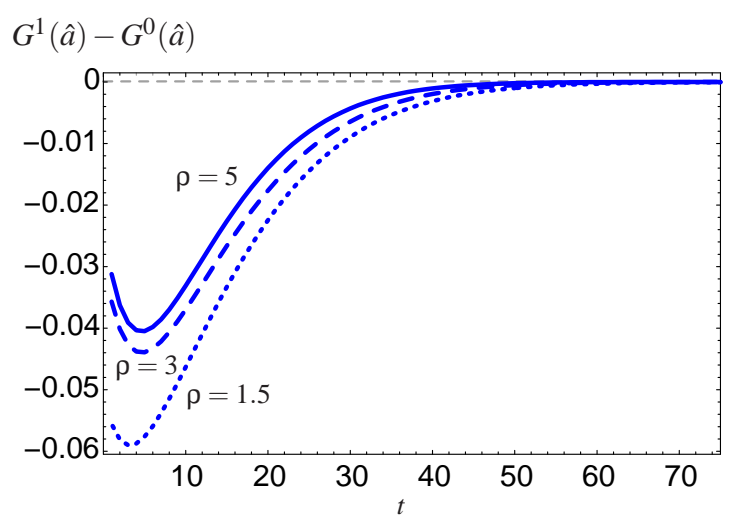

(b) Wealth inequality

Figure 3: Transitory adjustments of the growth rate and wealth inequality

rate even rises beyond the equilibrium interest rate (by (2), $r=0.03$ ) for sufficiently large values of the standard deviation of labor efficiency.

A larger dispersion in the idiosyncratic labor income shocks naturally raises income inequality. This also conveys into higher wealth inequality. The increase in wealth inequality is less pronounced for higher degrees of risk aversion, reflecting the related increase in inequality aversion associated with CRRA preferences and indicating the leveling effect of precautionary motives for individual asset holdings and intertemporal consumption smoothing.

Figure 3 displays the transitory dynamics of the growth rate and wealth inequality following an instantaneous doubling of income risk $(s d(\ln \theta)=0.3 \rightarrow 0.6)$. The values are given relative to the new growth equilibrium. We find that the transitory adjustment paths are non-monotonic for both quantities. Following an initial jump, the growth rate and the Gini coefficient of wealth display an interim drop, before both attain their corresponding higher new equilibrium levels in the long run. The drop is least pronounced for higher degrees of risk aversion. It is altogether small in magnitude, amounting at most to a tenth of a percentage point for the growth rate. The non-monotonic dynamics can be traced back to revisions in households' accumulation decisions and associated changes in the wealth distribution. Agents are unequally affected by a rise in risk. The poorer ones receive a larger fraction of household income from labor and therefore are more exposed to risk than the richer ones. Their immediate increase in savings explains the temporarily more equal distribution of wealth before the overall rise in risk dominates in the long run and the wealth distribution converges towards its new stationary limit which is characterized by higher inequality.

We now turn towards the welfare effects of a change in risk. As outlined above, the welfare measure (14) expresses welfare gains (losses) as the aggregate percentage change in consumption necessary to keep each and all agents indifferent between the original and the new situation. Figure 4 plots the welfare measure against the standard deviation of the labor efficiency shock. As before, we provide results for three different degrees of 
Figure 4: Welfare and labor income risk

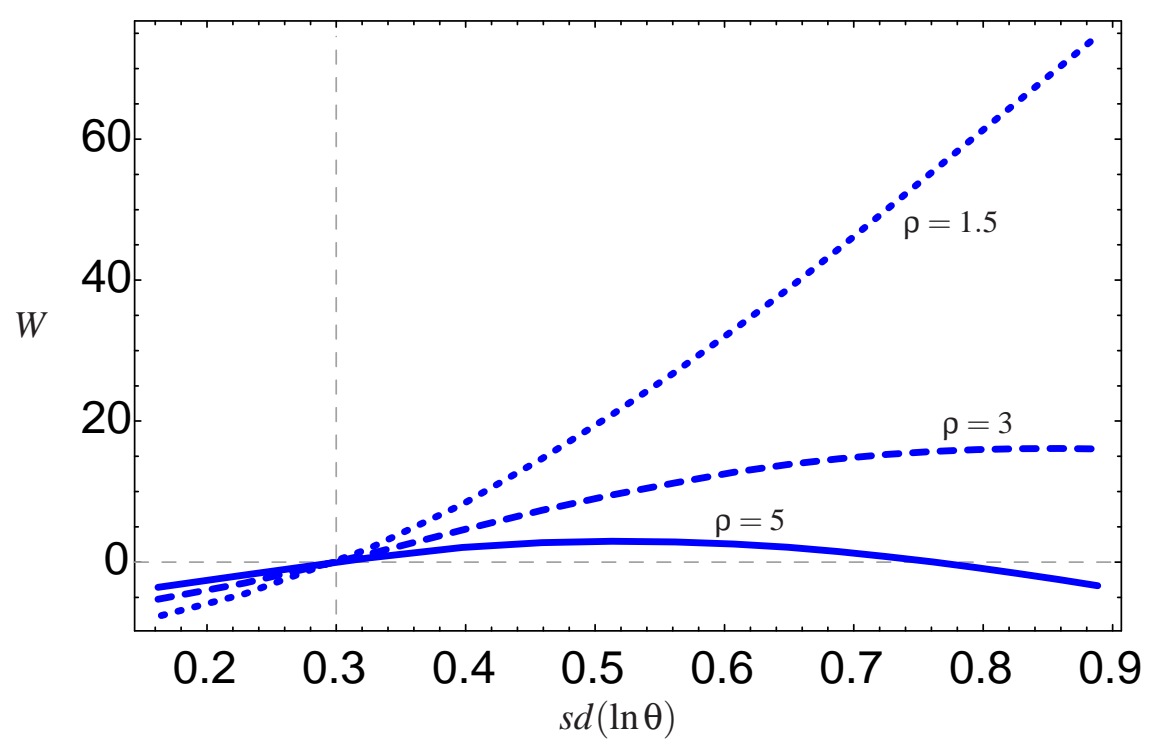

risk aversion. The vertical dashed line indicates the baseline model as starting point for welfare comparisons, where $s d(\ln \theta)=0.3$.

We find that the relationship between idiosyncratic risk and welfare is non-monotonic and inverted-U shaped. ${ }^{10}$ Compared to the standard neoclassical incomplete markets model, welfare gains (and losses) in our model result from a combination of two, a direct and an indirect effect associated with a rise in risk. The direct effect is well-known and straightforward, with welfare unambiguously decreasing for higher levels of risk due to concavity of the underlying utility function. The indirect effect is related to the positive change in the equilibrium growth rate of the economy. A rise in risk enforces bufferstock saving, which translates into higher growth and hence higher welfare. By increasing savings, households not only self-insure against fluctuations in their intertemporal consumption profile but also shift additional resources into the riskless income source. The positive growth effect first dominates in the determination of welfare, but finally the risk effect takes over and welfare declines for higher levels of risk.

In terms of the results presented in Figure 4 for $\rho=5$, moving from $s d(\ln \theta)=0.3$ to $s d(\ln \theta)=0.9$ amounts to an equivalent welfare loss resulting from a five percent decrease in aggregate consumption. The overall welfare effect is more pronounced for lower degrees of risk aversion. Consider the case of $\rho=3$. Although the positive growth effect is smaller in magnitude too (see Figure 2a), this more than compensates for the negative welfare effect from increased risk, such that altogether, the welfare effect from switching between the baseline scenario and, say for instance, $s d(\ln \theta)=0.9$ is larger.

Given that insurance and credit markets are incomplete, we identify a tradeoff relationship between risk and growth in the determination of welfare. A cautious interpreta-

\footnotetext{
${ }^{10}$ This is also true for the lower degree of risk aversion, $\rho=1.5$, where the turning points are associated with levels of risk outside the range depicted in figure 4.
} 
tion of our results suggests that a policy targeted at the reduction of risk not necessarily generates welfare improvements.

To illustrate this issue, consider a thought experiment, where the government offers agents a perpetual and complete elimination of individual income risks from time $t$ onward, e.g., by taxing away labor incomes and redistributing them in a lump-sum fashion with a transfer equal to mean wage income $\tilde{w}$. The immediate effect of providing full insurance against idiosyncratic risk would be that the stationary wealth distribution is frozen and perpetually fixed in its time- $t$ state. The economy immediately jumps onto a new balanced growth path, where the equilibrium growth rate is given by the value it attains under the complete markets regime, $\gamma_{C}=(\beta R)^{1 / \rho}$.

By Proposition 1, equilibrium growth from time $t$ onward will now be lower than before under the incomplete markets regime, because the importance of holding buffer-stocks has ceased. Hence, insurance comes at the cost of lower growth. Individuals have to weigh the utility gain from eliminating idiosyncratic risk against the welfare losses stemming from the reduction in the aggregate growth rate. The redistributive policy reduces the risk associated with after-tax labor incomes. The resulting negative growth effect from a decline in savings can probably dominate the positive effect from lower risk. It turns out that aggregate support for such a redistributive policy crucially depends on who benefits most from this policy. Whereas relatively rich agents are comparably unconcerned about the reduction in labor income risk, the relatively poor ones benefit most. ${ }^{11}$

Welfare from time $t$ onward of a household with wealth $\hat{a}$ under the redistributive regime can be determined explicitly as: ${ }^{12}$

$$
V_{R}(\hat{a})=\frac{1}{1-\tilde{\beta}} \frac{\left(\left(R-\left(1+\gamma_{C}\right)\right) \hat{a}+\tilde{w}\right)^{1-\rho}}{1-\rho} .
$$

Table 2 lists our numerical results and provides information on the decline in the growth rate measured in percentage points, on the population fraction of supporters, who would actually like to have this policy implemented, and on the aggregate welfare gain (or loss respectively). The equilibrium growth rate is reduced by roughly half a percentage point, which amounts to a welfare loss equal to an almost 6-9\% reduction in aggregate consumption. The negative growth effect is larger for higher degrees of risk aversion, as the precautionary motive is more pronounced here.

Because the positive insurance effect of redistribution only outweighs the negative growth effect for the lowest income classes, at most one fifth to one forth of the population would support implementation of perfect risk-pooling. This indicates that the opportunity costs of insurance in terms of forgone growth are substantial for large fractions of the

\footnotetext{
${ }^{11}$ In a similar spirit, Krueger and Perri (2011) discuss crowding out effects of public insurance in the standard incomplete markets model but abstract from potential distortions on capital accumulation and redistributive consequences. In our model, public insurance directly crowds out growth.

${ }^{12}$ The expression for optimal consumption follows from the intertemporal budget constraint, by utilizing the condition that $\hat{a}^{\prime}=\hat{a}$ in steady-state and rearranging.
} 
Table 2: Perfect risk-pooling and welfare

\begin{tabular}{ccr|ccr}
\hline \hline \multicolumn{3}{c|}{$\rho=3$} & \multicolumn{4}{c}{$\rho=5$} \\
\hline p.p. change in $\gamma$ & $\%$ in favor & $W$ & p.p. change in $\gamma$ & $\%$ in favor & $W$ \\
\hline-0.43 & 17.77 & -8.01 & -0.56 & 25.54 & -5.78 \\
\hline \hline
\end{tabular}

Table 3: Debt limits, growth and inequality $(\rho=3)$

\begin{tabular}{c|ccc}
\hline \hline$\tilde{\phi}$ & $\gamma$ & $G(\hat{a})$ & $G(\tilde{y})$ \\
\hline 0.00 & 0.0091 & 0.485 & 0.231 \\
0.50 & 0.0078 & 0.643 & 0.280 \\
1.00 & 0.0072 & 0.742 & 0.328 \\
2.00 & 0.0067 & 0.841 & 0.412 \\
3.00 & 0.0064 & 0.883 & 0.474 \\
4.00 & 0.0063 & 0.902 & 0.512 \\
4.935 & 0.0062 & 0.911 & 0.536 \\
\hline \hline
\end{tabular}

population. The long-run negative consequences for growth tend to dominate the positive ones of providing ex post insurance in the determination of welfare.

\subsection{Debt Limits and Growth}

So far, we have assumed an ad hoc debt limit of zero in our numerical simulations. We now relax this assumption. By allowing for nonzero debt limits, we examine the growth and distributional effects of raising ad hoc debt limits up to the natural debt limit (7), which reflects the endogenously determined upper bound beyond which no household would be willing to increase debt. Given the Markov-chain approximation of the $A R(1)$ process for the baseline parameterization of Table 1, we determine the lowest possible realization for labor efficiency as $\theta_{\min }=0.52475$. The associated natural debt limit can then be determined as $\tilde{\phi}_{n}=4.935$, which amounts to almost five times detrended average wealth (see also Definition 1). Pushing the ad hoc debt limit further beyond this limit implies that the natural debt limit is binding by (8), and the associated growth rate is bounded from above by the real interest rate as stated in Proposition 1.

Knowing that a narrow interpretation of our model theoretically implies labor incomes to be lognormally distributed and hence a $\theta_{\min }$ infinitesimally close to naught, for the sake of the argument, we momentarily would like to interpret our approximation as describing the 'true' process driving labor productivity. From this we expect some more general insights into the question of how borrowing constraints affect growth and inequality.

The results are reported in Table 3 for the case of $\rho=3$. The simulation results extend our conclusions for the two cases $\tilde{\phi}_{n}$ and $\tilde{\phi}_{a h}=0$ drawn earlier in section 2.3 and illustrated in Figure 1. Tightening borrowing constraints $(\tilde{\phi} \rightarrow 0)$ has a positive impact on growth. The risk averse agents increase wealth holdings, which solely reflects an effect in buffer- 
stock saving, because the equilibrium interest rate is not affected by the magnitude of debt limits. Intuitively, higher debt limits allow households to 'stretch' their budget constraint by allowing for a higher level of consumption to be financed from debt. The demand for intertemporal self-insurance is comparably smaller.

Table 3 shows that the overall growth effect of tightening borrowing constraints is rather modest from a quantitative viewpoint. For instance, doubling the borrowing limit from $\tilde{\phi}=1$ to $\tilde{\phi}=2$ only lowers the equilibrium growth rate by $1 / 20$ of a percentage point. Looking at the entire range of admissible borrowing limits, the difference in equilibrium growth rates is less than one third of a percentage point.

Regarding the distributional consequences we find that relaxing borrowing constraints has a marked effect on income and wealth inequality. The results are reported in Table 3. One implication of generally permitting nonzero debt limits is that we observe negative wealth holdings under the stationary distribution. The reported Gini coefficients are normalized as described in Chen et al. (1982) to take account of these negative wealth and income levels. The Gini of wealth rises from 0.48 to 0.91 over the range of admissible debt limits. Of course, individual income is more equally distributed than individual wealth, but still the Gini rises from 0.23 to 0.53 .

\section{Growth Policy}

\subsection{Policy Implications}

The policy implications of the Romer (1986)-model are well-known. Due to the production externality, the private return to capital falls short of the social return, and households save less than the socially optimal amount. The optimal policy provides an incentive to save more by subsidizing accumulation and closing the wedge between the private and the social return. Let $s$ denote a subsidy on the factor price of capital. An optimal policy $\bar{s}$ completely internalizes the knowledge spillover, establishes the Pareto-efficient allocation, and consequently is welfare-maximizing in an economy with complete markets. The optimal subsidy relates to the relative magnitude of the externality and is determined by the partial elasticities of production, $\bar{s}=(1-\alpha) / \alpha$.

Another standard implication following from the theory of optimal taxation is that tax revenues necessary to balance the public budget in this context should be raised in a nondistortionary fashion, i.e. by a lump-sum tax if available, or-in the case of inelastic labor supply—a consumption tax or a labor income tax.

The outlined policy recommendations and results are valid for an economy populated by homogenous agents. If household are heterogeneous regarding their relative factor endowments, this is also true for the individual-related income shares accruing from the two income sources. Beyond that, factor incomes in our model also differ with respect to the risk involved. Due to the presence of borrowing constraints, agents choose different saving rates. Consequently, benefits and burdens of the underlying policy are not likely 
to be equally distributed. This issue was raised by Bertola (1993), who considered the Arrow/Romer-type economy with exogenously given and time-invariant wealth heterogeneity and pointed out that the optimal policy financed from a labor income tax is not a likely outcome of a majority vote, if the median voter has relative endowment holdings below the mean. Originally brought forward by Meltzer and Richard (1981), we expect this result to remain valid in a growing economy with an endogenously determined wealth distribution. A policy which is optimal in the complete markets economy not necessarily is welfare-maximizing in a heterogeneous-agent economy and also not likely to have support from a majority of agents.

The general policy implication of providing households with an additional incentive to accumulate is preserved in our model, but it is also necessary to shed more light on the growth, redistributive, and welfare effects of financing the subsidy payments. In this context, we have to account for several dimensions. Firstly, a subsidy affects the riskiness of after-tax income, thereby possibly providing an insurance against the individual income risk. Secondly, as far as wealth levels and the endogenously determined wealth distribution are concerned, the underlying policy may possibly interact with the extent to which borrowing constraints are actually binding. A chosen policy gives rise to primary and secondary effects on the aggregate growth rate of the economy and on wealth inequality. Thirdly, a consumption tax tends to be regressive if saving rates increase in the level of income. The distribution of the associated welfare gains and losses across the population is not necessarily straightforward and will be one of the issues raised in our subsequent analysis.

We start our policy analysis with a simple growth policy, where the subsidy on capital returns is financed from revenues from a consumption tax, the latter known to be nondistortionary in an intertemporal context. Our numerical analysis offers results on growth, inequality and welfare for a change in the subsidy rate. Because the underlying policy sustains heterogeneity among households, we also discuss the implications for a politico-economic equilibrium in section 4.3.

\subsection{Growth Policy}

We assume that capital accumulation is subsidized at the rate $s$ paid on the factor price of capital. The post-subsidy real interest factor is then given by $R_{s}=R+s(R-1+\delta)$. Subsidy payments are financed out of revenues from a consumption tax $\tau$. We can write down the agents intertemporal budget constraint in detrended form as

$$
\hat{a}^{\prime}(1+\gamma)+\tilde{c}(1+\tau)=R_{s} \hat{a}+\left[(1+\gamma)-R_{s}\right] \tilde{\phi}+\tilde{w} \theta
$$

with $R_{S}$ simply replacing the no-policy interest factor $R$. The associated value function of the problem is equivalent to the one given in (6). 
Table 4: Growth, consumption, and consumption risk under policy $s, \tau$

\begin{tabular}{ll|ll|ll||ll|ll}
\hline \hline & & \multicolumn{4}{|c||}{$\rho=3.0$} & \multicolumn{4}{c}{$\rho=5.0$} \\
\hline \multirow{2}{*}{$s$} & & \multicolumn{2}{|c|}{ repr. agent } & \multicolumn{2}{c||}{ incompl. markets } & \multicolumn{3}{c}{ repr. agent } & \multicolumn{2}{|c}{ incompl. markets } \\
\cline { 3 - 11 } & $\gamma_{C}$ & $\tau$ & $\gamma$ & $\tau$ & $\gamma_{C}$ & $\tau$ & $\gamma$ & $\tau$ \\
\hline 0.0 & 0.03 & 0.0048 & 0.0 & 0.0091 & 0.0 & 0.0029 & 0.0 & 0.0085 & 0.0 \\
0.4 & 0.07 & 0.0189 & 0.19 & 0.0239 & 0.19 & 0.0113 & 0.19 & 0.0181 & 0.19 \\
0.8 & 0.12 & 0.0327 & 0.40 & 0.0380 & 0.41 & 0.0195 & 0.40 & 0.0267 & 0.39 \\
1.2 & 0.16 & 0.0460 & 0.64 & 0.0514 & 0.65 & 0.0274 & 0.64 & 0.0346 & 0.60 \\
1.6 & 0.21 & 0.0591 & 0.91 & 0.0644 & 0.93 & 0.0350 & 0.91 & 0.0420 & 0.83 \\
2.0 & 0.25 & 0.0718 & 1.21 & 0.0769 & 1.25 & 0.0425 & 1.21 & 0.0492 & 1.08 \\
2.4 & 0.29 & 0.0842 & 1.56 & 0.0892 & 1.61 & 0.0497 & 1.56 & 0.0561 & 1.48 \\
\hline \hline
\end{tabular}

The government budget is balanced in each period of time, if ${ }^{13}$

$$
\tau=\frac{s(R-1+\delta)}{R-(1+\gamma)+(1-\alpha) B} .
$$

The results of our numerical simulations are given in Tables 4 and 5. They are based on the calibration as outlined in the preceding section (see also Table 1). Again, we compare the allocation resulting under the ad hoc debt limit of $\tilde{\phi}_{a h}=0$ and the complete markets economy. For given subsidy rates, the table provides information on the consumption tax rate necessary to keep the public budget balanced, the equilibrium interest factor, and the resulting equilibrium growth rate of the economy for the two cases $\rho=3$ and $\rho=5$.

Starting from an equilibrium without policy intervention, we increase the subsidy rate in steps of 0.4. The optimal level of the subsidy in our numerical example which completely internalizes the knowledge spillover can be determined as $\bar{s}=(1-\alpha) / \alpha=2.03$. The associated growth rate of the complete markets economy is given by $\gamma_{C, \bar{s}}=0.0718$ for $\rho=3$ and $\gamma_{C, \bar{s}}=0.0425$ for $\rho=5$ respectively.

Naturally, the aggregate growth rate increases with a rise in the capital subsidy in all settings under consideration. The rise in the net interest rate provides an incentive to increase individual savings. Adjustments in the equilibrium net interest rate follow the rise in the subsidy, the initial interest rate rising by more than factor eight for subsidy rates close to $\bar{s}^{14}$ The positive growth effect is most pronounced in the complete markets economy, the rise in the growth rate amounting to 3/4 of the associated increase in $s$ (highest vs lowest nonzero value of $s$ ). The growth effect is smaller for the incomplete markets economies under consideration, amounting to $3 / 5$ of the associated increase in $s$ for the case of $\rho=3$ and $1 / 2$ for $\rho=5$.

\footnotetext{
${ }^{13}$ Total public revenues and spendings are derived by aggregating individual budget constraints in equilibrium, such that $\hat{A}(1+\gamma)=R_{S} \hat{A}+\left[(1+\gamma)-R_{S}\right] \tilde{\phi}+\tilde{w}-(1+\tau) \tilde{C}$. Aggregate subsidy payments equal $s(R-1+\delta) \tilde{A}$, and tax revenues are given by $\left.\tau \tilde{C}=\frac{\tau}{1+\tau}\left(\left[R_{S}-(1+\gamma)\right] \tilde{A}\right)+\tilde{w}\right)$. With $\hat{A}-\tilde{\phi}=\tilde{A}=1$ in equilibrium, a balanced budget requires $s(R-1+\delta) \tilde{A}=\tau \tilde{C}$ which implies (16).

${ }^{14}$ Given the parameterization of production technology, this outcome is standard for this class of endogenous growth models.
} 
Table 5: Growth and inequality under policy $s, \tau$

\begin{tabular}{l|lll||lll}
\hline \hline \multirow{3}{*}{$s$} & \multicolumn{3}{|c||}{$\rho=3.0$} & \multicolumn{3}{c}{$\rho=5.0$} \\
\cline { 2 - 7 } & $\gamma$ & $G(\hat{a})$ & $G(\tilde{y})$ & $\gamma$ & $G(\hat{a})$ & $G(\tilde{y})$ \\
\hline 0.0 & 0.0091 & 0.485 & 0.231 & 0.0029 & 0.445 & 0.222 \\
0.4 & 0.0239 & 0.479 & 0.251 & 0.0113 & 0.439 & 0.237 \\
0.8 & 0.0380 & 0.459 & 0.261 & 0.0195 & 0.409 & 0.240 \\
1.2 & 0.0514 & 0.435 & 0.264 & 0.0274 & 0.379 & 0.238 \\
1.6 & 0.0644 & 0.411 & 0.263 & 0.0350 & 0.354 & 0.234 \\
2.0 & 0.0769 & 0.391 & 0.261 & 0.0425 & 0.331 & 0.229 \\
2.4 & 0.0892 & 0.372 & 0.258 & 0.0497 & 0.312 & 0.223 \\
\hline \hline
\end{tabular}

The general result stated in Proposition 1 that growth under idiosyncratic uninsurable risk exceeds growth in the complete markets economy is reflected in our numerical simulations for all values of $s$. The policy scheme under consideration indirectly lowers the riskiness of individual total income by raising the relative income share accruing to (riskless) individual capital income, which becomes obvious if we consider the budget constraint (15). The lower income risk generates a disincentive to save out of precautionary motives and explains the comparably smaller growth effect in the incomplete markets economy.

The Gini coefficient of income displays a non-monotonic pattern for a rise in $s$, first rising and declining again for higher subsidies; see Table 5 . Contrary, wealth inequality is declining monotonically for rising $s$. The subsidy induced increase in accumulation exerts a leveling effect on the wealth distribution. Indirectly, individual budget constraints are relaxed. This becomes obvious from the intertemporal budget constraint (15). Given our baseline specification $\tilde{\phi}_{a h}=0$, the household receives higher returns for a given wealth level.

Regarding the welfare effects of capital subsidization, it is important to note that the relatively rich benefit from it more than the relatively poor, firstly, because interest payments make up a larger share in their individual incomes and secondly, because the regressive consumption tax. Figure 5 illustrates the welfare effect of a growth policy targeted at internalizing the knowledge spillover in accumulation. As before, we calculate the welfare gain in terms of a utilitarian welfare measure, tracing back welfare gains to equivalent percentage increases in consumption, leaving each agent-and in the aggregate all agents-indifferent between the no-policy situation and the policy of having a subsidy of size $s$. For reference, we also display the complete markets economy (dashed lines). The figure shows that welfare effects are smaller in magnitude in the incomplete markets economy.

Of course, the maximum welfare gain in the complete markets economy results for a subsidy of size $\bar{s}=(1-\alpha) / \alpha$ which completely closes the wedge between the private and the social return to capital. The welfare-maximizing policy under incomplete mar- 


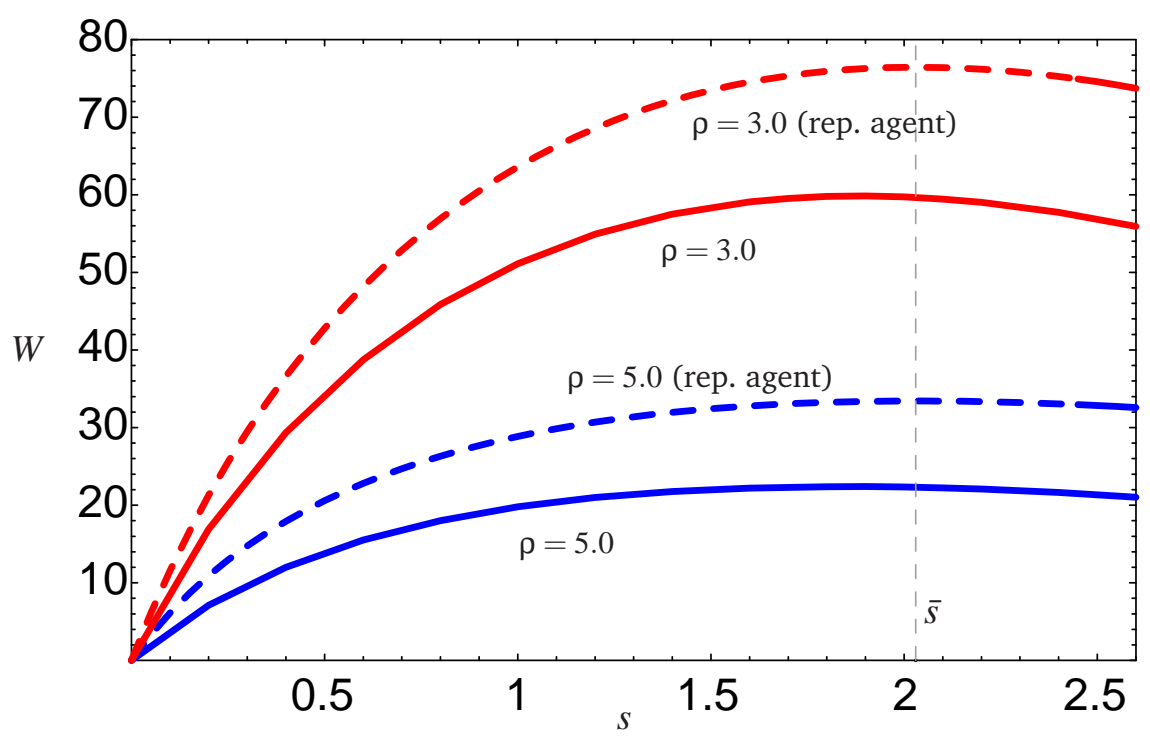

Figure 5: Welfare and optimal growth policy

kets is characterized by a smaller degree of subsidization because of the regressive effect of taxing consumption, which hurts poor agents comparably stronger. Summing up, a benevolent social planner seeking to maximize aggregate welfare would choose a policy which does not completely internalize the external effect in capital accumulation, thus balancing welfare gains and losses over the entire population. Another major implication of the policy analysis is the negative correlation between growth and wealth inequality. The underlying policy also has a leveling effect on the distribution of wealth.

\subsection{Politico-economic Equilibrium}

The welfare statements of the preceding section regarding the issue of optimality rely on a utilitarian welfare measure, where we first determine the compensating variation for each household, then aggregate and last solve for the welfare-maximizing growth policy. This does, however, not necessarily imply that the optimal policy indeed will be voted into effect in a majority vote. The reason is that agents do not symmetrically benefit from capital subsidization. Inequality of income and wealth determines the political equilibrium. Those, who draw a larger income share from the accumulating factor will benefit more from an investment subsidy than the relatively capital-poor. This was already acknowledged by Bertola (1993) who found for an exogenously given invariant wealth distribution that policies which focus on income redistribution across reproducible and non-reproducible factors tend to slow down growth the more the stronger the political power of those who own only relatively small amounts of the accumulating factor.

The politico-economic implications of our approach are quite similar to Bertola's findings. But moreover, as growth and inequality both are endogenous in the underlying model, this adds another channel through which policy affects the equilibrium allocation. 


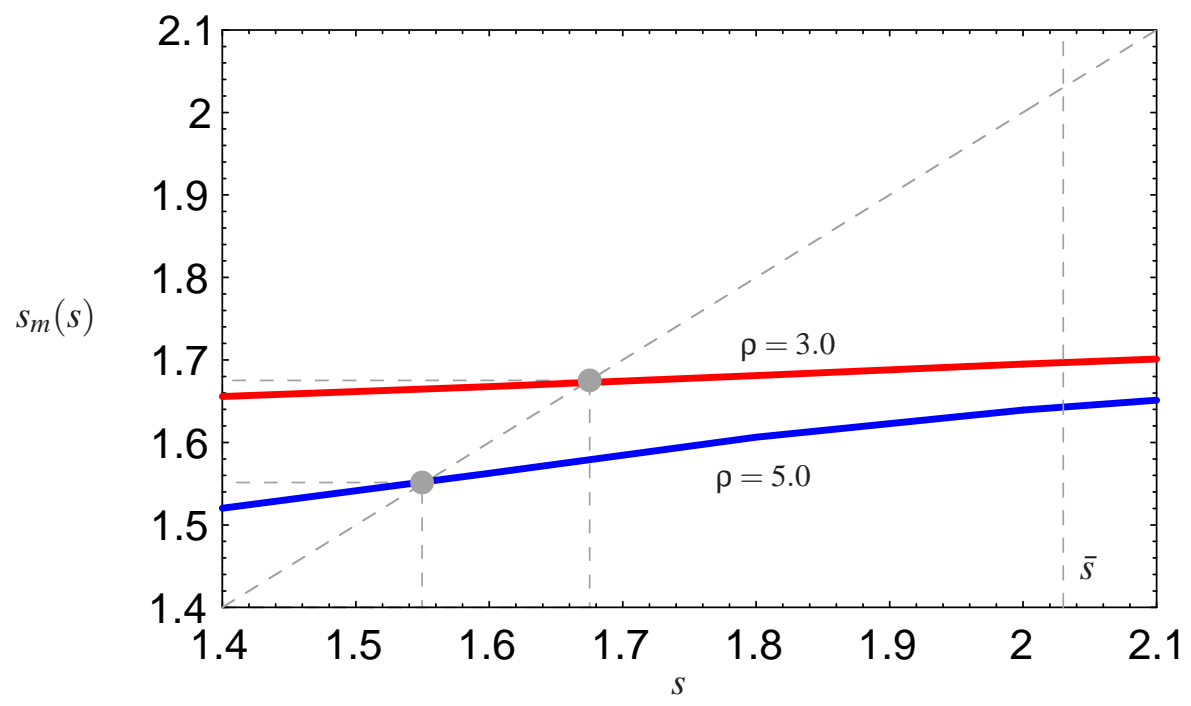

Figure 6: Politico-economic equilibrium

In our model, the equilibrium growth rate resulting from the voted policy also affects the equilibrium distribution of wealth. Table 5 shows that for rising rates of capital subsidization the higher growth rates are accompanied by less wealth inequality, and we do not observe the commonly posited tradeoff between those two.

We determine the politico-economic equilibrium by first solving the model numerically for different levels of the subsidy $s$ consistent with a balanced public budget. This provides us with the necessary information on the consumers' individual preferences towards alternative levels of subsidization. Let $V(\hat{a}, \theta ; s)$ denote the value function under policy s. $V(\hat{a}, \theta ; s)$ is single-peaked in $s$, such that there exists a unique individually preferred subsidy for each given combination of individual wealth $\hat{a}$ and productivity $\theta$.

In a next step we compute the cumulative density $\Psi\left(s^{*} ; s\right)$ of individually preferred subsidies $s^{*}$ over households having productivity $\theta$ and owning wealth $\hat{a}$ by using the stationary distribution $\mu$ at a given policy $s$. Let $s_{m}^{*}(s)$ denote the subsidy preferred by the median voter, i.e. $\Psi\left(s_{m}^{*} ; s\right)=0.5$. To determine the politico-economic equilibrium, we finally iterate over $s$ to find a level $s_{m}^{*}$ such that $\Psi\left(s_{m}^{*} ; s_{m}^{*}\right)=0.5$.

Figure 6 plots the function $s_{m}^{*}(s)$ for the two alternative degrees of risk aversion under consideration. The associated politico-economic equilibria are represented by the intersection of the function $s_{m}^{*}(s)$ with the $45^{\circ}-$ line. Because the underlying stochastic process for labor efficiency generates a wealth distribution which is skewed to the left, the median voter prefers a subsidy which falls short of the optimal one maximizing aggregate welfare, $s_{m}^{*}<\bar{s}$. The knowledge spillover is only incompletely internalized, and there remains a wedge between the private and the social return to capital. The associated equilibrium growth rate is lower in the politico-economic equilibrium, and the wealth distribution remains more unequal; see also Table 5 . The equilibrium subsidy is the lower the more 
risk averse (or likewise inequality averse) consumers are, yielding values of $s_{m}^{*}=1.68$ for $\rho=3$ and $s_{m}^{*}=1.55$ for $\rho=5$.

\section{Conclusion}

In this paper we combined the neoclassical standard incomplete markets model with idiosyncratic risks and borrowing constraints in the spirit of Aiyagari $(1994,1995)$ with a simple growth mechanism, namely the learning-by-doing approach by Romer (1986). A special feature of our approach is that both, the equilibrium income and wealth distribution as well as the long-run equilibrium growth rate, are endogenously determined in our model. We therefore are able to study possible feedback effects between risk, growth, and inequality and to discuss the redistributive and growth implications of public policy. To this end our approach aims at a qualitative and quantitative assessment of the consequences of market imperfections on long-run growth and inequality in order to gain a better understanding of the subject, rather than being viewed as modeling an actual economy, calibrated and simulated to match certain empirical regularities.

We derive necessary and sufficient conditions for the existence of a balanced growth path and demonstrate that these not necessarily have to be met, such that an equilibrium growth path possibly might not exist under a given numerical specification of the model.

As well known from the literature, the presence of uninsurable risk and borrowing constraints unambiguously has a positive effect on the long-run growth rate of the economy. Endogenous growth in our model stems from human capital externalities. This endogenous growth mechanism is known to generate allocations which fail Pareto-efficiency. Our numerical simulations come up with some interesting results for the interaction between growth, risk, and inequality. We find that a policy aimed at pushing growth by subsidizing interest payments (financed from a non-distortionary consumption tax) also tends to reduce the observed wealth inequality. Contrary, a redistributive policy aimed at completely eliminating the individual income risk stands at odds with the growth target and may turn out welfare-deteriorating in the whole. Moreover, the policy under consideration also lowers the riskiness of disposable income, such that feedback effects on intertemporal accumulation decisions and growth have to be taken into account.

This is also important, when it comes to the evaluation of welfare effects. Generally, welfare gains are directly related to either lower risk or higher growth. Lower risk, however, is accompanied by a decrease in aggregate savings and consequently a decline in the equilibrium growth rate. Depending on the magnitude of risk, one of the effects is offsetting the other, such that we observe an inverted U-shaped relationship between welfare and risk.

The aggregate welfare implications of the underlying policy in the representative agent growth model are no longer valid in the growing standard incomplete markets economy. Depending on their individual wealth level, welfare gains from the subsidy are unequally distributed across the society, while the consumption tax exerts a regressive effect. Alto- 
gether, this amounts to a smaller welfare-maximizing level of subsidization compared to the complete markets economy and is also reflected in the outcome of a majority vote over alternative public policies. We find that the median voter prefers less than optimal subsidies on investment. Interestingly, a majority might even vote against a policy providing full insurance, because welfare losses due to lower growth more than offset welfare gains from having lower risk.

\section{References}

Aghion, Philippe, Caroli, Eve, and García-Peñalosa, Cecilia (1999), Inequality and Growth: The Perspective of the New Growth Theories, Journal of Economic Literature, 37, 1615-1660.

Aiyagari, S. Rao (1994), Uninsured Idiosyncratic Risk and Aggregate Saving, Quarterly Journal of Economics, 109 (3), 659-684.

- (1995), Optimal Capital Income Taxation with Incomplete Markets, Borrowing Constraints, and Constant Discounting, Journal of Political Economy, 103 (6), 1158-1175.

Aiyagari, S. Rao and McGrattan, Ellen R. (1998), The Optimum Quantity of Debt, Journal of Monetary Economics, 42, 447-469.

Banerjee, Abhijit V. and Newman, Andrew F. (1993), Occupational Choice and the Process of Development, Journal of Political Economy, 101, 274-298.

Bénabou, Roland (1996), Inequality and Growth, in: Ben S. Bernanke and Julio J. Rotemberg (eds.), NBER Macroeconomics Annual, MIT Press, Cambridge, MA, vol. 11, pp. $11-74$.

- (2000), Unequal Societies: Income Distribution and the Social Contract, American Economic Review, 90 (1), 96-129.

Bertola, Giuseppe (1993), Factor Shares and Savings in Endogenous Growth, American Economic Review, 83, 1185-1198.

Bertola, Guiseppe, Foellmi, Reto, and Zweimüller, Peter (2006), Income Distribution in Macroeconomic Models, Princeton University Press, Princeton, NJ.

Bewley, Truman (1983), A Difficulty with the Optimum Quantity of Money, Econometrica, 51 (5), 1485-1504.

Carroll, Christopher D. (1997), Buffer-Stock Saving and the Life Cycle/Permanent Income Hypothesis, Quarterly Journal of Economics, 112 (1), 1-56.

Chen, Chau-Nan, Tsaur, Tien-Wang, and Rhai, Tong-Shieng (1982), The Gini Coefficient and Negative Income, Oxford Economic Papers, pp. 473-478.

Domeij, David and Heathcote, Jonathan (2004), On the Distributional Effects of Reducing Capital Taxes, International Economic Review, 45 (2), 523-554.

Galor, Oded and Zeira, Joseph (1993), Income Distribution and Macroeconomics, Review of Economic Studies, 60, 35-52. 
Guvenen, Fatih (2009), An Empirical Investigation of Labor Income Processes, Review of Economic Dynamics, 12, 58-79.

Heathcote, Jonathan (2005), Fiscal Policy with Heterogeneous Agents and Incomplete Marktes, Review of Economic Studies, 72, 161-188.

Huggett, Mark (1993), The Risk-Free Rate in Heterogeneous-Agent IncompleteInsurance Economies, Journal of Economic Dynamics and Control, 17 (5-6), 953-969.

Japelli, Tullio and Pagano, Marco (1994), Saving, Growth and Liquidity Constraints, Quarterly Journal of Economics, 109, 83-109.

- (1999), The Welfare Effects of Liquidity Constraints, Oxford Economic Papers, 51, 410 430.

Kopecky, K and Suen, R. (2010), Finite Markov-chain Approximations to Highly Persistent Processes, Review of Economic Dynamics, 13, 701-714.

Krebs, Tom (2003a), Growth and Welfare Effects of Business Cycles in Economies with Idiosyncratic Human Capital Risk, Review of Economic Dynamics, 6, 846-868.

- (2003b), Human Capital Risk and Economic Growth, Quarterly Journal of Economics, 118, 709-745.

Krueger, Dirk and Perri, Fabrizio (2011), Public versus Private Risk Sharing, Journal of Economic Theory, 146, 920-956.

Meltzer, Allan H. and Richard, Scott F. (1981), A Rational Theory of the Size of Government, Journal of Political Economy, 89, 914-927.

Prescott, Edward C. (1986), Theory ahead of Business-Cycle Measurement, CarnegieRochester Conference Series on Public Policy, 25, 11-44.

Romer, Paul M. (1986), Increasing Returns and Long-Run Growth, Journal of Political Economy, 94, 1002-1037.

Rouwenhorst, K. Geert (1995), Asset Pricing Implications of Equilibrium Business Cycle Models, in: Thomas F. Cooley (ed.), Frontiers of Business Cycle Research, Princeton University Press, Princeton, pp. 294-330.

Storesletten, Kjetil, Telmer, Chris I., and Yaron, Amir (2004), Cyclical Dynamics in Idiosyncratic Labor Market Risk, Journal of Political Economy, 112 (3), 695-717.

\section{A Appendix}

\section{A.1 Proof of 1. and 2. of Proposition 1}

We reformulate problem (6) by defining and substituting $\bar{c}=\tilde{c} /(1+\gamma), \bar{R}=R /(1+\gamma), \bar{w}=\tilde{w} /(1+\gamma)$ :

$$
\begin{aligned}
V(\hat{a}, \theta) & =\max _{\bar{c}, \hat{a}^{\prime} \geqslant 0}\left\{(1+\gamma)^{1-\rho} u(\bar{c})+\tilde{\beta} \mathrm{E}_{\theta}^{\prime} V\left(\hat{a}^{\prime}, \theta^{\prime}\right)\right\} \\
\text { s.t. } \quad \hat{a}^{\prime}+\bar{c} & =\bar{R} \hat{a}+[1-\bar{R}] \tilde{\phi}+\bar{w} \theta
\end{aligned}
$$


Reformulating the optimization problem this way does not affect optimal decisions, which when aggregated — are represented by the function $\tilde{A}(\gamma, \tilde{\phi}, R, \tilde{w})$, describing aggregate (detrended) wealth holdings for given $\gamma, \tilde{\phi}, R$ and $\tilde{w}$. Given the reformulated problem (A.1), the solution properties and proofs provided by Aiyagari (1994) for the case of iid shocks straightforwardly carry over to the present model. As already outlined in the text, we are not able to provide a formal proof of existence for serially correlated shocks, but verify existence in our numerical analysis.

We furthermore have to specify an ad hoc debt limit $\tilde{\phi}_{a h} \geqslant 0$, whenever $\bar{R} \leqslant 1$ for a proper formulation of the optimization problem (A.1). For $\bar{R}>1$, a natural debt limit $\tilde{\phi}_{n}$ might be binding before any ad hoc limit takes effect. The natural debt limit is given by:

$$
\tilde{\phi}_{n}=\frac{\bar{w} \theta_{\min }}{\bar{R}-1}
$$

where $\theta_{\min }$ denotes the lowest possible realization of $\theta$. We rewrite condition (8) as:

$$
\tilde{\phi}= \begin{cases}\tilde{\phi}_{a h}, & \bar{R} \leqslant 1 \\ \min \left[\tilde{\phi}_{a h}, \tilde{\phi}_{n}\right], & \bar{R}>1\end{cases}
$$

Upper and lower bounds on feasible growth rates:

Problem (A.1) is bounded only if $\tilde{\beta}<1$. This implies the following restrictions on feasible growth rates for $\rho>0, \rho \gtrless 1$ :

$$
\begin{array}{ll}
1+\gamma<\beta^{\frac{1}{\rho-1}}, & \rho<1 \\
1+\gamma>\beta^{\frac{1}{\rho-1}}, & \rho>1 .
\end{array}
$$

Depending on the size of $\rho$, these restrictions impose upper and lower bounds on feasible growth rates. For $\rho=1, \tilde{\beta}=\beta$, and feasibility is met by assumption.

An important feature of the function $\tilde{A}(\gamma, \tilde{\phi}, \tilde{w}, R)$ is that aggregate (detrended) wealth holdings rise to infinity as $\bar{R}$ approaches $\tilde{\beta}$ from below (see Aiyagari, 1994). Given the definitions of $\bar{R}$ and $\tilde{\beta}, \tilde{\beta} \bar{R}<1$ is equivalent to:

$$
1+\gamma>(\beta R)^{1 / \rho}=1+\gamma_{C},
$$

which proves that the equilibrium growth rate under incomplete markets and idiosyncratic risk exceeds the equilibrium growth rate $\gamma_{C}$ under complete markets.

Properties of $\tilde{A}(\gamma, \tilde{\phi}, \tilde{w}, R)$ :

Regarding detrended wealth holdings, we can state the following: For any ad hoc debt limit $\tilde{\phi}_{a h} \geqslant 0$, the function $\tilde{A}(\gamma, \tilde{\phi}, \tilde{w}, R) \rightarrow-\tilde{\phi}$ if $\bar{R} \rightarrow 0$ from above, which with $R$ fixed by technology follows from $1+\gamma \rightarrow \infty$. For $\tilde{\phi}_{n}, \bar{R}>1$ (i.e. $r>\gamma$ ) by (A.3). As $\tilde{\phi}_{n} \rightarrow \infty$ for $\bar{R} \rightarrow 1$ from above, by (A.2), we obtain $\tilde{A}(\gamma, \tilde{\phi}, \tilde{w}, R) \rightarrow-\infty$.

Combining (A.4) and (A.5) implies the following:

(i) $\rho>1$ : Whenever the lower bound given by (A.4) is lower than $1+\gamma_{C}$, the function $\tilde{A}(\gamma, \tilde{\phi}, \tilde{w}, R)$ is well defined for all $\gamma>\gamma_{C}$. The respective condition is equivalent to $\beta<R^{\rho-1}$, and given the above described properties of the function $\tilde{A}(\gamma, \tilde{\phi}, R, \tilde{w})$, this conditions turns out to be sufficient for existence of a balanced growth path, i.e. an equilibrium growth rate $\gamma>\gamma_{C}$ such that $\tilde{A}(\gamma, \tilde{\phi}, \tilde{w}, R)=1$. $\gamma$ is bounded from above by $r$ in case of a natural debt limit, which follows from the properties of the function $\tilde{A}(\gamma, \tilde{\phi}, \tilde{w}, R)$. 
(ii) $\rho=1$ : This case is identical to (i) except that there are no additional restriction on feasible growth rates. As the function $\tilde{A}(\gamma, \tilde{\phi}, R, \tilde{w})$ is well defined for all $\gamma>\gamma_{C}$, existence of a balanced path follows immediately. Condition $\beta<R^{\rho-1}$ becomes $\beta<1$ if $\rho=1$ and is satisfied by assumption.

(iii) $\rho<1$ : Whenever the growth rate is smaller than the upper bound implied by (A.4), the function $\tilde{A}(\gamma, \tilde{\phi}, R, \tilde{w})$ is well defined for $\gamma>\gamma_{C}$. Thus, a necessary condition for existence of a balanced growth path is that $1+\gamma>\beta^{\frac{1}{\rho-1}}>1+\gamma_{C}$, which is equivalent to $\beta<R^{\rho-1}$. Given the properties of $\tilde{A}(\gamma, \tilde{\phi}, \tilde{w}, R)$, the balanced growth path exists in case of a natural debt limit if the upper bound is greater than or equal to $r$. The condition $1+\gamma>\beta^{\frac{1}{\rho-1}} \geqslant 1+r$ is equivalent to $\beta \leqslant R^{\rho-1}$. Thus, a balanced growth path with $r>\gamma>\gamma_{C}$ such that $\tilde{A}(\gamma, \tilde{\phi}, \tilde{w}, R)=1$ exists in case of a natural debt limit, if $\beta<R^{\rho-1}$. In case of an ad hoc debt limit, $\tilde{A}(\gamma, \tilde{\phi}, \tilde{w}, R)$ decreases from $+\infty$ as $\gamma$ rises above $\gamma_{C}$. However, it might be that the upper bound implied by (A.4) is hit before an equilibrium is attained. Therefore, the condition $\beta<R^{\rho-1}$ is only necessary for existence of a balanced growth path in case of $\rho<1$.

Proof completed.

\section{A.2 Illustration of nonexistence of a balanced growth equilibrium in the case $\rho<1$}

Figure 7: Nonexistence of a balanced growth path with $\rho=0.52$ and $\tilde{\phi}_{a h}=0$

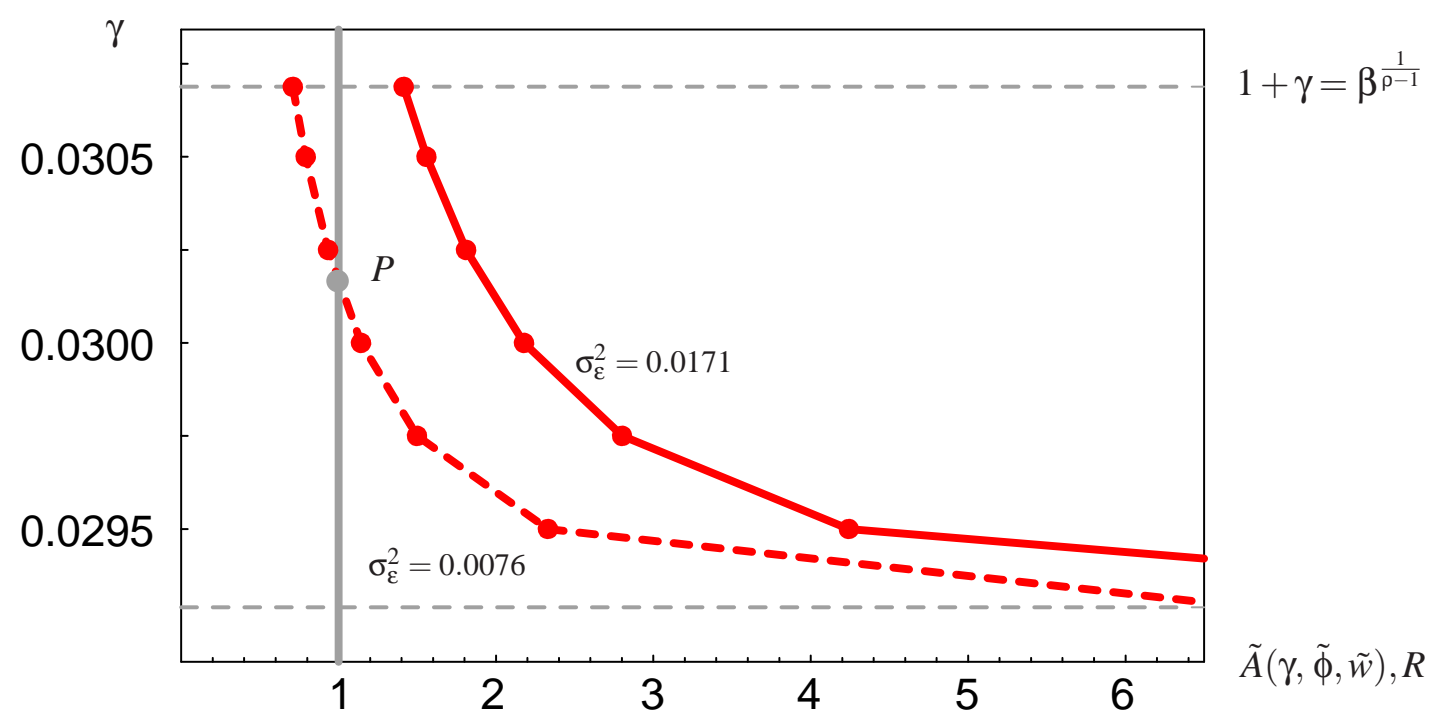

Given that all other parameters of the model are specified as in Table 1, the necessary condition for existence of an equilibrium $\left(\beta<R^{\rho-1}\right)$ implies that a balanced growth path will not exist, whenever $\rho<1+\ln \beta / \ln R \approx 0.488508$. As $\beta<R^{\rho-1}$ is only necessary but not sufficient in the presence of an ad hoc debt limit, a balanced growth path might not exist here, even if $\rho$ exceeds this value.

Figure 7 shows the possibility of such an outcome, by using results from numerical simulations of the model assuming a value of $\rho=0.52$ and an ad hoc debt limit $\tilde{\phi}_{a h}=0$. The resulting growth rate of the complete markets economy is given by $\gamma_{C}=0.02815$ and, according to (A.4), the upper bound on growth can be determined as $\gamma=0.031988$. Aggregate (detrended) asset holdings 
Figure 8: Transitory dynamics of the capital stock

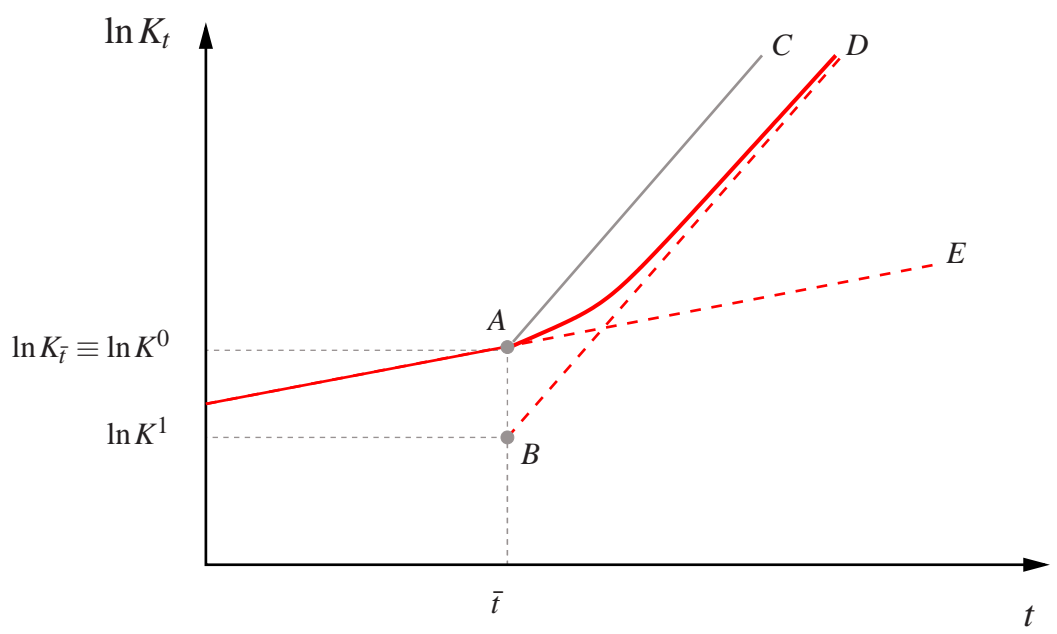

$\tilde{A}(\gamma, \tilde{\phi}, R, \tilde{w})$ are represented by the solid line, which decreases monotonically as $\gamma$ moves from the lower bound $\gamma_{C}$ towards the upper bound, but remain strictly above the value $\tilde{A}(\gamma, \tilde{\phi}, \tilde{w}, R)=1$, indicating the capital market equilibrium. Consequently, the balanced growth equilibrium does not exist within the lower and upper bound of feasible growth rates (at the upper bound for $\gamma$ our simulations return a wealth level of $\tilde{A}=1.494$ ).

Just for illustration, compare this result to a situation associated with lower idiosyncratic risk $\left(\sigma_{\varepsilon}^{2}=0.0076\right.$, dashed curve in Figure 7). Lower values of $\sigma_{\varepsilon}^{2}$ shift the asset holding curve to the left, now implying existence of a growth equilibrium in Point $P$.

\section{B Transitional Dynamics}

\section{B.1 Technical issues}

We develop a procedure allowing us to calculate the actual time path of the capital stock relative to an artificially constructed 'reference path'. Following a once and for all change in the economic environment, the reference path mimics the identical long-run evolution of the capital stock of the original economy under transitional dynamics, but posits instead that the economy has grown at the new equilibrium (constant) growth rate from the outset, while starting from a lower initial level.

Figure 8 provides a graphical illustration of the actual time path of capital and the associated reference path. As of time $\bar{t}$, the economy of the complete markets model would immediately jump onto a new equilibrium growth path, represented by the line segment $A C$ and reflecting an increase in the growth rate. Compared to this, the transitory dynamics of the incomplete markets economy can be described by the curve $A D$. The actual time path of capital asymptotically approaches a path with a constant growth rate as described by the line $B D$. In what follows, this will be referred to as 'reference path'. The associated initial value $K^{1}$ for the capital stock on this reference path differs from the original capital stock $K^{0}$ present in period $\bar{t}$ when the change in the environmental conditions occurs.

We assume that the new equilibrium growth rate $\gamma$, the initial value for capital on the reference path $K^{1}$ as well as the complete transitional path $\left\{K_{\bar{t}+j}\right\}_{j=0}^{\infty}$ of the original capital stock from period 
$\bar{t}$ onwards are known. The capital stock along the reference path evolves according to $K^{1}(1+\gamma)^{j}$. The ratio of the two capital stocks along their respective paths for any given period $j=0,1, \ldots$ can then be expressed as:

$$
\lambda_{j}=\frac{K_{\bar{t}+j}}{K^{1}(1+\gamma)^{j}},
$$

where $\lambda_{0}=K^{0} / K^{1}$ by assumption.

In the course of detrending (see section 2.2), all macroeconomic variables are divided by the level of the aggregate capital stock on the reference path. This transformation of variables implies that the individual budget constraint for any period $\bar{t}+j$ during the phase of transition is given by:

$$
\hat{a}^{\prime}(1+\gamma)+\tilde{c}=R \hat{a}+[(1+\gamma)-R] \tilde{\phi}+\lambda_{j} \tilde{w} \theta, \quad j=0,1, \ldots
$$

Note that the wage rate in $\bar{t}+j$ is given $w_{\bar{t}+j}=\tilde{w} K_{\bar{t}+j}$ and the underlying transformations demand the division of $w_{\bar{t}+j}$ by $K^{1}(1+\gamma)^{j}$.

It is now possible to write down the sequence of optimization problems the agents solve throughout transition:

$$
\begin{aligned}
V_{j}(\hat{a}, \theta) & =\max _{\hat{a}^{\prime}}\left\{u(\tilde{c})+\tilde{\beta} \mathrm{E}_{\theta^{\prime}}\left[V_{j+1}\left(\hat{a}^{\prime}, \theta^{\prime}\right)\right]\right\} \quad j=0,1, \ldots \\
\text { s.t. } \quad \hat{a}^{\prime}(1+\gamma)+\tilde{c} & =R \hat{a}+[(1+\gamma)-R] \tilde{\phi}+\lambda_{j} \tilde{w} \theta \\
\lambda_{0} & =\frac{K^{0}}{K^{1}}
\end{aligned}
$$

\section{B.2 Computation of transitional dynamics}

Let $\mu^{0}(\hat{a}, \theta)$ denote the stationary distribution of agents across wealth and productivity levels along the old balanced growth path and $V_{*}^{1}(\hat{a}, \theta)$ the value function that solves the dynamic optimization problem along the new balanced growth path. The algorithm to compute the transition towards the new steady-state (and thereby the initial level of the new reference path) proceeds as follows:

1) Fix the number of periods $T$ for the transition phase and normalize the level of the capital stock in the period $\bar{t}$ where the transition starts to one (i.e. $K^{0}=1$ ).

2) Fix for $j=0, \ldots, T-1$ an initial sequence of weights $\lambda_{j}$ that describe the deviation of the capital stock from the new reference path during the transition. Set $\lambda_{0}=1$ and $\lambda_{T-1}=1$. Note, that $\lambda_{0}=1$ implies that the initial value for the new reference path is assumed to be $K^{1}=K^{0}=1$ (a natural choice is $\lambda_{j}=1$ for $j=1, \ldots, T-2$ ).

3) Starting with the value function $V_{*}^{1}(\hat{a}, \theta)$ for period $\bar{t}+T$ solve the dynamic optimization problem (A.6) iteratively backwards in time for $j=T-1, \ldots, 0$. This results in policy functions $h_{\bar{t}+j}(\hat{a}, \theta)$ for each $j=0, \ldots, T-1$.

4) Transform the stationary distribution $\mu^{0}(\hat{a}, \theta)$ such that it represents the stationary distribution given the new reference path. Let $\mu_{\bar{t}}^{1}(\hat{a}, \theta)$ denote this transformed distribution, then:

$$
\mu_{\bar{t}}^{1}(\hat{a}, \theta)=\mu^{0}\left(\hat{a} K^{1}, \theta\right)
$$

5) Given this transformed stationary distribution and the policy functions $h_{\bar{t}+j}(\hat{a}, \theta)$ for $j=$ $0, \ldots, T-1$, compute the distributions of agents across wealth and productivity levels during the transition $\mu_{\bar{t}+j+1}^{1}(\hat{a}, \theta)$. From this compute the value $\tilde{A}_{\bar{t}+j}$ of the transformed aggregate stock of capital for $j=0, \ldots, T-1$ :

$$
\tilde{A}_{\bar{t}+j}=\int_{\theta} \int_{\hat{a}} \mu_{\bar{t}+j}^{1}(\hat{a}, \theta) \mathrm{d} \hat{a} \mathrm{~d} \theta
$$


6) Use the deviation of $\tilde{A}_{\bar{t}+j}$ from the presupposed value $\lambda_{\bar{t}+j}$ for each $j=0, \ldots, T-1$ to update the initial guess in the following way (here $\varepsilon_{K}$ and $\varepsilon_{\lambda}$ are some small numbers that govern the speed of the updating process)

$$
\begin{aligned}
K^{\prime 1} & =K^{1}+\varepsilon_{K} \times\left(\tilde{A}_{\bar{t}+T-1}-1\right) \\
\mu_{\bar{t}}^{1}(\hat{a}, \theta) & =\mu^{0}\left(\hat{a} / K^{1^{\prime}}, \theta\right) \\
\lambda^{\prime} & =\int_{\theta} \int_{\hat{a}} \mu_{\bar{t}}^{1}(\hat{a}, \theta) \mathrm{d} \hat{a} \mathrm{~d} \theta \\
\lambda^{\prime}{ }_{j} & =\lambda_{j}+\varepsilon_{\lambda} \times\left(\tilde{A}_{\bar{t}+j}-\lambda_{j}\right), \quad j=1, \ldots, T-2
\end{aligned}
$$

and reenter into the iterative process at step 3) above until the deviations computed at step 6) are sufficiently small.

\section{B.3 Welfare effects including transitory dynamics}

Let $J(a, \theta)$ denote lifetime utility of an agent who today has wealth $a$ and labor productivity $\theta$. With $c_{t}$ denoting consumption of this agent for $t=0,1, \ldots$, we then get:

$$
J(a, \theta)=\mathrm{E}_{0} \sum_{t=0}^{\infty} \beta^{t} u\left(c_{t}\right)
$$

Assume that the respective reference path for capital is given by $K_{t}=K(1+\gamma)^{t}$ for $t=0, \ldots$. Then, using the transformation $\hat{c}_{t}=c_{t} / K_{t}$ as well as $\hat{a}_{t}=a_{t} / K_{t}$ and because of homotheticity of the underlying utility function, we get along a balanced growth path:

$$
J(a, \theta)=K^{1-\rho} \mathrm{E}_{0} \sum_{t=0}^{\infty} \tilde{\beta}^{t} u(\tilde{c})=K^{1-\rho} V_{*}(a / K, \theta),
$$

where $V_{*}$ is the value function solving the individual agent's optimization problem in the respective balanced growth equilibrium.

Now, let $V^{1}(\hat{a}, \theta)$ denote the value function resulting from the iterative procedure outlined above for period $\bar{t}$ (i.e. $j=0$ ) and let $K^{1}$ denote the associated initial level of capital on the reference path. The value function represents the maximum lifetime utility attained after a parameter change in period $\bar{t}$. Furthermore, let $V_{*}^{0}(\hat{a}, \theta)$ denote the respective value function associated with the original balanced growth path. Lifetime utility of an agent with wealth $a=\hat{a} K^{j}, j=0,1$ before and after the change can then be expressed as (using the normalization $K^{0}=1$ ):

$$
\begin{aligned}
& J^{0}(a, \theta)=V_{*}^{0}(a, \theta) \\
& J^{1}(a, \theta)=\left(K^{1}\right)^{1-\rho} V^{1}\left(a / K^{1}, \theta\right)
\end{aligned}
$$

Thus, the proportional increase in consumption $\Delta(a, \theta)$ necessary to make this individual indifferent between these two settings results as:

$$
\Delta(a, \theta)=K^{1}\left(\frac{V^{1}\left(a / K^{1}, \theta\right)}{V_{*}^{0}(a, \theta)}\right)^{\frac{1}{1-\rho}}-1
$$

